\title{
Analytic Solutions of Moment Partial Differential Equations with Constant Coefficients
}

\author{
By \\ Sławomir Michalik \\ (Cardinal Stefan Wyszyński University, Poland)
}

\begin{abstract}
We consider the Cauchy problem for linear moment partial differential equations with constant coefficients in two complex variables. We construct an integral representation of the solution of this problem and study its analyticity. As a result we derive a characterisation of multisummable formal solutions of the Cauchy problem.

Key Words and Phrases. Linear PDEs with constant coefficients, Formal power series, Moment functions, Moment PDEs, $k$-summability, Multisummability.

2010 Mathematics Subject Classification Numbers. 35C10, 35C15, $35 \mathrm{E} 15$.
\end{abstract}

\section{Introduction}

We study the initial value problem for a general linear moment partial differential equation with constant coefficients in two complex variables $t, z$

$$
P\left(\partial_{m_{1}, t}, \partial_{m_{2}, z}\right) u(t, z)=0, \quad \partial_{m_{1}, t}^{j} u(0, z)=\varphi_{j}(z) \quad(j=0, \ldots, n-1),
$$

where $P(\lambda, \zeta)$ is a polynomial in variables $(\lambda, \zeta)$ of degree $n$ with respect to $\lambda$, $\partial_{m_{1}, t}$ and $\partial_{m_{2}, z}$ denote the formal moment differentiations, and the Cauchy data $\varphi_{j}(z)$ are analytic functions in a complex neighbourhood of the origin.

The formal $m$-moment differentiation $\partial_{m, z}$ was introduced recently by Balser and Yoshino [8] as the linear operator on the space of power series defined by

$$
\partial_{m, z}\left(\sum_{j=0}^{\infty} \frac{u_{j} z^{j}}{m(j)}\right):=\sum_{j=0}^{\infty} \frac{u_{j+1} z^{j}}{m(j)},
$$

where $m(u)$ is a moment function (see Definitions 5 and 6).

This concept generalises the usual and fractional differentiation. Indeed, for $m(u)=\Gamma(1+u)$ the operator $\partial_{m, z}$ coincides with the usual differentiation $\partial_{z}$. Hence for $m_{1}(u)=m_{2}(u)=\Gamma(1+u),(1)$ is the initial value problem for a linear partial differential equation with constant coefficients. Moreover, for $p \in N$ and $m(u)=\Gamma(1+u / p)$ the operator $\partial_{m, z}$ is closely related to the $1 / p$ fractional differentiation $\partial_{z}^{1 / p}$ (see Remark 3). 
Such a general approach to PDEs is especially useful in the theory of $k$ summability, since the formal power series $\hat{u}(t, z)=\sum_{j=0}^{\infty} u_{j}(z) t^{j} / m_{1}(j)$ satisfies (1) if and only if its $k$-Borel transform $v(s, z)=\sum_{j=0}^{\infty} u_{j}(z) s^{j} /\left(m_{1}(j) \Gamma(1+j / k)\right)$ satisfies the same equation with the $m_{1}$-moment differentiation $\partial_{m_{1}, t}$ replaced by the $\tilde{m}_{1}$-moment differentiation $\partial_{\tilde{m}_{1}, s}$, where $\tilde{m}_{1}(u)=m_{1}(u) \Gamma(1+u / k)$. In that way the question about summability of formal solution of (1) is reduced to the question about analytic continuation properties of solution of the same equation with $m_{1}(u)$ replaced by $\tilde{m}_{1}(u)$. For that reason we are concerned with the study of analytic continuation properties of solutions to general moment partial differential equations.

In the paper we construct a formal solution $\hat{u}$ of (1) and study its Gevrey asymptotic properties. In the case when the formal solution $\hat{u}$ is convergent, its sum $u$ is an analytic solution of (1) defined in a complex neighbourhood of the origin. The main result establishes the relation between analytic continuation properties of $u$ and the Cauchy data $\varphi_{j}(j=0, \ldots, n-1)$. As a corollary we characterise the multisummable formal solution of (1) in terms of analytic continuation properties and growth estimates of the Cauchy data.

We proceed as follows. We represent $P(\lambda, \zeta)$ in the form

$$
P(\lambda, \zeta)=P_{0}(\zeta)\left(\lambda-\lambda_{1}(\zeta)\right)^{n_{1}} \ldots\left(\lambda-\lambda_{l}(\zeta)\right)^{n_{l}},
$$

where $P_{0}(\zeta)$ is a polynomial and $\lambda_{1}(\zeta), \ldots, \lambda_{l}(\zeta)$ are the roots of the characteristic equation $P(\lambda, \zeta)=0$ of (1) with multiplicities $n_{1}, \ldots, n_{l}\left(n_{1}+\cdots+n_{l}=n\right)$ respectively. However a formal solution of (1) may be not uniquely defined. To avoid this inconvenience, we choose the normalised formal solution $\hat{u}$, which satisfies also

$$
\left(\partial_{m_{1}, t}-\lambda_{1}\left(\partial_{m_{2}, z}\right)\right)^{n_{1}} \ldots\left(\partial_{m_{1}, t}-\lambda_{l}\left(\partial_{m_{2}, z}\right)\right)^{n_{l}} \hat{u}=0,
$$

where $\lambda_{1}\left(\partial_{m_{2}, z}\right), \ldots, \lambda_{l}\left(\partial_{m_{2}, z}\right)$ are the moment pseudodifferential operators (see Definition 8).

Next we show that $\hat{u}=\sum_{\alpha=1}^{l} \sum_{\beta=1}^{n_{\alpha}} \hat{u}_{\alpha \beta}$ with $\hat{u}_{\alpha \beta}$ being the formal solution of

$$
\left(\partial_{m_{1}, t}-\lambda_{\alpha}\left(\partial_{m_{2}, z}\right)\right)^{\beta} \hat{u}_{\alpha \beta}=0 .
$$

We prove that the Gevrey order of $\hat{u}_{\alpha \beta}$ depends on the order $q_{\alpha}$ of the pole of $\lambda_{\alpha}(\zeta)$ at infinity and depends on the orders $1 / k_{1}, 1 / k_{2}$ of moment functions $m_{1}$, $m_{2}$ respectively.

In the case when $1 / k_{1}=q_{\alpha} / k_{2}$, the formal solution $\hat{u}_{\alpha \beta}$ of (2) is convergent. Hence its sum $u_{\alpha \beta}$ is an analytic solution of (2) defined in a complex neighbourhood of the origin. Using an integral representation of solution $u_{\alpha \beta}$ we find the connection between analytic continuation properties of $u_{\alpha \beta}$ and the Cauchy data. 
In the case when $1 / k_{1}<q_{\alpha} / k_{2}$, we characterise $\left(q_{\alpha} / k_{2}-1 / k_{1}\right)^{-1}$-summable solutions of (2) in terms of analytic continuation properties of the Cauchy data.

Finally, returning to the formal solution $\hat{u}$ of (1), we describe multisummable solutions of $(1)$ in terms of $\varphi_{j}(j=0, \ldots, n-1)$. We also give the necessary corrections of our previous papers $[15,16,17]$ concerning summability of formal solutions of linear (fractional) PDEs.

In the last section the above results are extended to the inhomogeneous moment equations

$$
P\left(\partial_{m_{1}, t}, \partial_{m_{2}, z}\right) \hat{u}=\hat{f}, \quad \partial_{m_{1}, t}^{j} \hat{u}(0, z)=\varphi_{j}(z) \quad \text { for } j=0, \ldots, n-1,
$$

where the inhomogeneity $\hat{f}(t, z)$ is a formal power series with respect to $t$.

In general, a formal solution of (3) may also be not unique, but it is uniquely determined by every formal power series $\hat{g}$ satisfying $P_{0}\left(\partial_{m_{2}, z}\right) \hat{g}=\hat{f}$, since there is exactly one formal solution of (3) satisfying also the moment pseudodifferential equation

$$
\left(\partial_{m_{1}, t}-\lambda_{1}\left(\partial_{m_{2}, z}\right)\right)^{n_{1}} \ldots\left(\partial_{m_{1}, t}-\lambda_{l}\left(\partial_{m_{2}, z}\right)\right)^{n_{l}} \hat{u}=\hat{g} .
$$

We find the formal solution $\hat{u}$ of (3) determined by $\hat{g}$. As in the homogeneous case we show that $\hat{u}=\sum_{\alpha=1}^{l} \sum_{\beta=1}^{n_{\alpha}} \hat{u}_{\alpha \beta}$, where $\hat{u}_{\alpha \beta}$ satisfies

$$
\left(\partial_{m_{1}, t}-\lambda_{\alpha}\left(\partial_{m_{2}, z}\right)\right)^{\beta} \hat{u}_{\alpha \beta}=\hat{g}_{\alpha \beta}
$$

for some formal series $\hat{g}_{\alpha \beta}$ connected with $\hat{g}$. Expressing the formal solution $\hat{u}_{\alpha \beta}$ in terms of $\hat{g}$, we calculate the Gevrey order of $\hat{u}_{\alpha \beta}$ and we get the characterisation of analytic continuation properties and summability of $\hat{u}_{\alpha \beta}$ in terms of $\hat{g}$.

The present paper is a generalisation of $[15,17]$, where the characterisation of multisummable solutions of homogeneous and inhomogeneous linear PDEs with constant coefficients was given. The inspiration for our study was the paper of Balser and Yoshino [8], where the notion of moment differentiation was introduced and the Gevrey order of formal solutions of general inhomogeneous linear moment PDEs with constant coefficients was determined. Finally, let us point out that the summability of formal solutions of homogeneous linear PDEs with constant coefficients was studied by Balser [1, 3], Balser and Miyake [7], Ichinobe [9], Lutz, Miyake and Schäfke [10], Malek [12], Michalik [13, 15, 16] and Miyake [19]. The inhomogeneous case was investigated by Balser [4], Balser and Loday-Richaud [6], Balser, Duval and Malek [5] and Michalik [14, 17]. Moreover, Lysik [11] used the results of Lutz et al. [10] to characterise summable solutions of the Burgers equation.

\section{Notation, Gevrey formal power series and $k$-summability}

We use the following notation. The complex disc in $\boldsymbol{C}^{n}$ with centre at the origin and radius $r>0$ is denoted by $D_{r}^{n}:=\left\{z \in C^{n}:|z|<r\right\}$. To simplify 
notation, we write $D_{r}$ instead of $D_{r}^{1}$. If the radius $r$ is not essential, then we denote it briefly by $D^{n}$ (resp. $D$ ).

$A$ sector in a direction $d \in \boldsymbol{R}$ with an opening $\varepsilon>0$ in the universal covering space $\tilde{\boldsymbol{C}}$ of $\boldsymbol{C} \backslash\{0\}$ is defined by

$$
S_{d}(\varepsilon):=\left\{z \in \tilde{\boldsymbol{C}}: z=r e^{i \theta}, d-\varepsilon / 2<\theta<d+\varepsilon / 2, r>0\right\} .
$$

Moreover, if the value of opening angle $\varepsilon$ is not essential, then we denote it briefly by $S_{d}$. We denote by $\hat{S}_{d}\left(\operatorname{resp} . \hat{S}_{d}(\varepsilon)\right)$ the set $S_{d} \cup D\left(\right.$ resp. $\left.S_{d}(\varepsilon) \cup D\right)$.

By $\mathcal{O}(G)$ we understand the space of analytic functions on a domain $G \subseteq C^{n}$. The Banach space of analytic functions on $D_{r}$, continuous on its closure and equipped with the norm $\|\varphi\|_{r}:=\max _{|z| \leq r}|\varphi(z)|$ is denoted by $\boldsymbol{E}(r)$.

The space of formal power series

$$
\hat{u}(t, z)=\sum_{j=0}^{\infty} u_{j}(z) t^{j} \quad \text { with } u_{j}(z) \in \boldsymbol{E}(r)
$$

is denoted by $\boldsymbol{E}(r)[[t]]$. Moreover, we set $\boldsymbol{E}[[t]]:=\bigcup_{r>0} \boldsymbol{E}(r)[[t]]$.

In this section we also recall some definitions and fundamental facts about the Gevrey formal power series and $k$-summability. For more details we refer the reader to [2].

Definition 1. A function $u \in \mathcal{O}\left(S_{d}(\varepsilon) \times D_{r}\right) \quad\left(\right.$ resp. $\left.u \in \mathcal{O}\left(\hat{S}_{d}(\varepsilon) \times D_{r}\right)\right)$ is of exponential growth of order at most $K>0$ as $t \rightarrow \infty$ in $S_{d}(\varepsilon)$ (resp. in $\left.\hat{S}_{d}(\varepsilon)\right)$ if for any $r_{1} \in(0, r)$ and any $\varepsilon_{1} \in(0, \varepsilon)$ there exist $A, B<\infty$ such that

$$
\max _{|z| \leq r_{1}}|u(t, z)|<A e^{B|t|^{K}} \quad \text { for every } t \in S_{d}\left(\varepsilon_{1}\right) \quad\left(\text { resp. } t \in \hat{S}_{d}\left(\varepsilon_{1}\right)\right)
$$

The space of such functions is denoted by $\mathcal{O}^{K}\left(S_{d}(\varepsilon) \times D_{r}\right)$ (resp. $\left.\mathcal{O}^{K}\left(\hat{S}_{d}(\varepsilon) \times D_{r}\right)\right)$.

Analogously, a function $\varphi \in \mathcal{O}\left(S_{d}(\varepsilon)\right)$ (resp. $\left.\varphi \in \mathcal{O}\left(\hat{S}_{d}(\varepsilon)\right)\right)$ is of exponential growth of order at most $K>0$ as $z \rightarrow \infty$ in $S_{d}(\varepsilon)$ (resp. in $\hat{S}_{d}(\varepsilon)$ ) if for any $\varepsilon_{1} \in(0, \varepsilon)$ there exist $A, B<\infty$ such that

$$
\left.|\varphi(z)|<A e^{B|z|^{K}} \quad \text { for every } z \in S_{d}\left(\varepsilon_{1}\right) \text { (resp. } t \in \hat{S}_{d}\left(\varepsilon_{1}\right)\right) \text {. }
$$

The space of such functions is denoted by $\mathcal{O}^{K}\left(S_{d}(\varepsilon)\right)$ (resp. $\left.\mathcal{O}^{K}\left(\hat{S}_{d}(\varepsilon)\right)\right)$.

Definition 2. Let $s \geq 0$. A formal power series

$$
\hat{u}(t, z):=\sum_{j=0}^{\infty} u_{j}(z) t^{j} \quad \text { with } u_{j}(z) \in \boldsymbol{E}(r)
$$


is called a Gevrey formal power series in $t$ of order $s$ if its coefficients satisfy

$$
\max _{|z| \leq r}\left|u_{j}(z)\right| \leq A B^{j} \Gamma(1+s j) \quad \text { for } j=0,1, \ldots
$$

with some positive constants $A$ and $B$.

The set of Gevrey formal power series in $t$ of order $s$ over $\boldsymbol{E}(r)$ is denoted by $\boldsymbol{E}(r)[[t]]_{s}$. We also set $\boldsymbol{E}[[t]]_{s}:=\bigcup_{r>0} \boldsymbol{E}(r)[[t]]_{s}$.

Definition 3. Let $k>0$ and $d \in \boldsymbol{R}$. A formal series $\hat{u} \in \boldsymbol{E}[[t]]_{1 / k}$ defined by (4) is called $k$-summable in a direction $d$ if its $k$-Borel transform $v$ satisfies

$$
v(s, z):=\sum_{j=0}^{\infty} u_{j}(z) \frac{s^{j}}{\Gamma(1+j / k)} \in \mathcal{O}^{k}\left(\hat{S}_{d}(\varepsilon) \times D\right)
$$

for some $\varepsilon>0$.

The $k$-sum of $\hat{u}(t, z)$ in the direction $d$ is represented by the Laplace transform of $v$

$$
u^{\theta}(t, z):=\frac{1}{t^{k}} \int_{0}^{\infty(\theta)} e^{-(s / t)^{k}} v(s, z) d s^{k}
$$

where the integration is taken over any ray $e^{i \theta} \boldsymbol{R}_{+}:=\left\{r e^{i \theta}: r \geq 0\right\}$ with $\theta \in$ $(d-\varepsilon / 2, d+\varepsilon / 2)$.

We are now ready to define multisummability in some multidirection.

Definition 4. Let $k_{1}>\cdots>k_{n}>0$. We say that a real vector $\left(d_{1}, \ldots, d_{n}\right)$ $\in \boldsymbol{R}^{n}$ is an admissible multidirection with respect to $\left(k_{1}, \ldots, k_{n}\right)$ if

$$
\left|d_{j}-d_{j-1}\right| \leq \pi\left(1 / k_{j}-1 / k_{j-1}\right) / 2 \quad \text { for } j=2, \ldots, n .
$$

Let $\mathbf{k}=\left(k_{1}, \ldots, k_{n}\right) \in \boldsymbol{R}_{+}^{n}$ and let $\mathbf{d}=\left(d_{1}, \ldots, d_{n}\right) \in \boldsymbol{R}^{n}$ be an admissible multidirection. We say that a formal power series $\hat{u}$ is $\mathbf{k}$-summable in the multidirection $\mathbf{d}$ if $\hat{u}=\hat{u}_{1}+\cdots+\hat{u}_{n}$, where $\hat{u}_{j}$ is $k_{j}$-summable in the direction $d_{j}$ for $j=1, \ldots, n$.

\section{Moment methods}

In this section we recall the notion of moment summability methods introduced by Balser [2].

Definition 5 (see [2, Section 5.5]). A pair of functions $e_{m}(z)$ and $E_{m}(z)$ is said to be kernel functions of order $k(k>1 / 2)$ if they have the following properties: 
1. $e_{m}(z) \in \mathcal{O}\left(S_{0}(\pi / k)\right), e_{m}(z) / z$ is integrable at the origin, $e_{m}(x) \in \boldsymbol{R}_{+}$for $x \in \boldsymbol{R}_{+}$and $e_{m}(z)$ is exponentially flat of order $k$ in $S_{0}(\pi / k)$ (i.e. $\forall_{\varepsilon>0} \exists_{A, B>0}$ such that $\left|e_{m}(z)\right| \leq A e^{-(|z| / B)^{k}}$ for $\left.z \in S_{0}(\pi / k-\varepsilon)\right)$.

2. $E_{m}(z) \in \mathcal{O}^{k}(\boldsymbol{C})$ and $E_{m}(1 / z) / z$ is integrable at the origin in $S_{\pi}(2 \pi-\pi / k)$.

3. The connection between $e_{m}(z)$ and $E_{m}(z)$ is given by the corresponding moment function $m(u)$ of order $1 / k$ as follows. The function $m(u)$ is defined in terms of $e_{m}(z)$ by

$$
m(u):=\int_{0}^{\infty} x^{u-1} e_{m}(x) d x \quad \text { for } \operatorname{Re} u \geq 0
$$

and the kernel function $E_{m}(z)$ has the power series expansion

$$
E_{m}(z)=\sum_{j=0}^{\infty} \frac{z^{j}}{m(j)} \quad \text { for } z \in C .
$$

Observe that in case $k \leq 1 / 2$ the set $S_{\pi}(2 \pi-\pi / k)$ is not defined, so the second property in Definition 5 can not be satisfied. It means that we must define the kernel functions of order $k \leq 1 / 2$ and the corresponding moment functions in another way.

Definition 6 (see [2, Section 5.6]). A function $e_{m}(z)$ is called a kernel function of order $k>0$ if we can find a pair of kernel functions $e_{\tilde{m}}(z)$ and $E_{\tilde{m}}(z)$ of order $p k>1 / 2$ (for some $p \in N$ ) so that

$$
e_{m}(z)=e_{\tilde{m}}\left(z^{1 / p}\right) / p \quad \text { for } z \in S_{0}(\pi / k) .
$$

For a given kernel function $e_{m}(z)$ of order $k>0$ we define the corresponding moment function $m(u)$ of order $1 / k$ by $(5)$ and the kernel function $E_{m}(z)$ of order $k$ by (6).

Remark 1. Observe that by Definitions 5 and 6 we have

$$
m(u)=\tilde{m}(p u) \quad \text { and } \quad E_{m}(z)=\sum_{j=0}^{\infty} \frac{z^{j}}{m(j)}=\sum_{j=0}^{\infty} \frac{z^{j}}{\tilde{m}(j p)} .
$$

Remark 2 (see [2, Section 5.5]). If $m(u)$ is a moment function of order $1 / k$ then the moments $m(j)$ are of the same order as $\Gamma(1+j / k)$. It means that there exist constants $c, C>0$ such that

$$
c^{j} \Gamma(1+j / k) \leq m(j) \leq C^{j} \Gamma(1+j / k) \quad \text { for } j \in N .
$$

The most important examples of kernel functions of order $k>0$ with corresponding moment functions are given by 
- $e_{m}(z)=k z^{k} e^{-z^{k}}$

- $m(u)=\Gamma(1+u / k)$

- $E_{m}(z)=\sum_{j=0}^{\infty} z^{j} / \Gamma(1+j / k)=: \boldsymbol{E}_{1 / k}(z)$, where $\boldsymbol{E}_{1 / k}$ is the Mittag-Leffler function of index $1 / k$.

The next proposition provides a method for the construction of new moment functions.

Proposition 1 (see [2, Theorem 31]). Let two kernel functions $e_{m_{j}}(z)$ of orders $k_{j}$ with corresponding moment functions $m_{j}(u)(j=1,2)$ be given. Then there is a unique kernel function $e_{m}(z)$ of order $k=\left(1 / k_{1}+1 / k_{2}\right)^{-1}$ with corresponding moment function $m(u)=m_{1}(u) m_{2}(u)$.

By Remark 2 and by the general theory of moment summability (see [2, Section 6.5 and Theorem 38]) we may characterise the Gevrey order and the $k$-summability of formal power series as follows.

Proposition 2. Let $m(u)$ be a moment function of order $1 / k, \hat{u}(t, z)=$ $\sum_{n=0}^{\infty} u_{n}(z) t^{n}$ be a formal power series with coefficients $u_{n}(z) \in \mathcal{O}(D)$ and $v(s, z):=$ $\sum_{n=0}^{\infty} u_{n}(z) s^{n} / m(n)$. Then

- $\hat{u}$ is a Gevrey series of order $1 / k$ if and only if $v \in \mathcal{O}\left(D^{2}\right)$.

- $\hat{u}$ is $k$-summable in a direction $d(d \in \boldsymbol{R})$ if and only if $v \in \mathcal{O}^{k}\left(\hat{S}_{d} \times D\right)$.

\section{Moment operators}

In this section we recall the notion of moment differential operators constructed recently by Balser and Yoshino [8]. We also introduce the concept of moment pseudodifferential operators, which generalise the pseudodifferential operators defined in $[15,16]$.

Definition 7. For every moment function $m(u)$ the linear operator $\partial_{m, x}: \boldsymbol{E}[[x]] \rightarrow \boldsymbol{E}[[x]]$ defined by

$$
\partial_{m, x}\left(\sum_{j=0}^{\infty} \frac{u_{j}}{m(j)} x^{j}\right):=\sum_{j=0}^{\infty} \frac{u_{j+1}}{m(j)} x^{j}
$$

is called the moment differential operator $\partial_{m, x}$.

Moreover, the right-inversion operator $\partial_{m, x}^{-1}: \boldsymbol{E}[[x]] \rightarrow \boldsymbol{E}[[x]]$ given by

$$
\partial_{m, x}^{-1}\left(\sum_{j=0}^{\infty} \frac{u_{j}}{m(j)} x^{j}\right):=\sum_{j=1}^{\infty} \frac{u_{j-1}}{m(j)} x^{j}
$$

is called the moment integration operator $\partial_{m, x}^{-1}$. 
Remark 3. Observe that

- For $m(j)=\Gamma(1+j)$, the operator $\partial_{m, x}$ coincides with the usual differentiation $\partial_{x}$.

- For $m(j)=\Gamma(1+j / k)(k>0)$, the operator $\partial_{m, x}$ satisfies

$$
\left(\partial_{m, x} u\right)\left(x^{1 / k}\right)=\partial_{x}^{1 / k}\left(u\left(x^{1 / k}\right)\right),
$$

where $\partial_{x}^{1 / k}$ is the Caputo fractional derivative of order $1 / k$ (see also [17, Definition 5 and Remark 1]) defined by

$$
\partial_{x}^{1 / k}\left(\sum_{j=0}^{\infty} \frac{u_{j}}{\Gamma(1+j / k)} x^{j / k}\right):=\sum_{j=0}^{\infty} \frac{u_{j+1}}{\Gamma(1+j / k)} x^{j / k} .
$$

By the end of this section we assume that $e_{m}(z)$ and $E_{m}(z)$ are kernel functions of order $k>0$ with a corresponding moment function $m(u)$.

The moment differential operator $\partial_{m, z}$ is well-defined for every $\varphi \in \mathcal{O}(D)$. In addition, we have the following integral representation of $\partial_{m, z}^{n} \varphi$.

Proposition 3. Let $\varphi \in \mathcal{O}\left(D_{r}\right)$. Then for every $|z|<\varepsilon<r$ and $n \in \boldsymbol{N}$ we have

$$
\partial_{m, z}^{n} \varphi(z)=\frac{1}{2 \pi i} \oint_{|w|=\varepsilon} \varphi(w) \int_{0}^{\infty(\theta)} \zeta^{n} E_{m}(z \zeta) \frac{e_{m}(w \zeta)}{w \zeta} d \zeta d w
$$

where $\theta \in(-\arg w-\pi /(2 k),-\arg w+\pi /(2 k))$.

Proof. Since $\varphi \in \mathcal{O}\left(D_{r}\right)$, we see that

$$
\varphi(z)=\sum_{j=0}^{\infty} \frac{\varphi^{(j)}(0)}{j !} z^{j}=m(0) \sum_{j=0}^{\infty} \frac{\partial_{m, z}^{j} \varphi(0)}{m(j)} z^{j} \quad \text { for }|z|<r .
$$

Hence, by the Cauchy integral formula

$$
\partial_{m, z}^{j} \varphi(0)=\frac{m(j)}{j ! m(0)} \varphi^{(j)}(0)=\frac{m(j)}{2 \pi i m(0)} \oint_{|w|=\varepsilon} \frac{\varphi(w)}{w^{j+1}} d w \quad \text { for } \varepsilon<r .
$$

By the definition of moment functions we have

$$
\frac{m(j)}{w^{j+1}}=\int_{0}^{\infty} y^{j-1} \frac{e_{m}(y)}{w^{j+1}} d y \stackrel{y=\zeta w}{=} \int_{0}^{\infty(\theta)} \zeta^{j} \frac{e_{m}(\zeta w)}{\zeta w} d \zeta \quad \text { with } \theta=-\arg w .
$$

Moreover, since $e_{m}(z)$ is exponentially flat of order $k$ for $\arg z \in(-\pi /(2 k)$, $\pi /(2 k))$, we may replace the direction $\theta=-\arg w$ by any direction $\theta \in$ $(-\arg w-\pi /(2 k),-\arg w+\pi /(2 k))$. 
It means that

$$
\partial_{m, z}^{j} \varphi(0)=\frac{1}{2 \pi i m(0)} \oint_{|w|=\varepsilon} \varphi(w) \int_{0}^{\infty(\theta)} \zeta^{j} \frac{e_{m}(\zeta w)}{\zeta w} d \zeta d w
$$

and consequently for $|z|<\varepsilon$ we have

$$
\begin{aligned}
\varphi(z) & =m(0) \sum_{j=0}^{\infty} \frac{\partial_{m, z}^{j} \varphi(0)}{m(j)} z^{j}=\frac{1}{2 \pi i} \oint_{|w|=\varepsilon} \varphi(w) \int_{0}^{\infty(\theta)} \frac{e_{m}(\zeta w)}{\zeta w} \sum_{j=0}^{\infty} \frac{\zeta^{j} z^{j}}{m(j)} d \zeta d w \\
& =\frac{1}{2 \pi i} \oint_{|w|=\varepsilon} \varphi(w) \int_{0}^{\infty(\theta)} E_{m}(\zeta z) \frac{e_{m}(\zeta w)}{\zeta w} d \zeta d w .
\end{aligned}
$$

Since

$$
\partial_{m, z}^{n} E_{m}(\zeta z)=\partial_{m, z}^{n} \sum_{j=0}^{\infty} \frac{\zeta^{j} z^{j}}{m(j)}=\sum_{j=0}^{\infty} \frac{\zeta^{j+n} z^{j}}{m(j)}=\zeta^{n} E_{m}(\zeta z)
$$

we finally obtain (7).

The formula (7) motivates the introduction of moment pseudodifferential operators on the space of analytic functions. To this end, let $\lambda(\zeta)$ be an analytic function for $|\zeta| \geq r_{0}$ (for some $r_{0}>0$ ) of polynomial growth at infinity. By (8) we may define

$$
\lambda\left(\partial_{m, z}\right) E_{m}(\zeta z):=\lambda(\zeta) E_{m}(\zeta z) \quad \text { for }|\zeta| \geq r_{0} .
$$

Hence we have

Definition 8. A moment pseudodifferential operator $\lambda\left(\partial_{m, z}\right)$ is defined by

$$
\lambda\left(\partial_{m, z}\right) \varphi(z):=\frac{1}{2 \pi i} \oint_{|w|=\varepsilon} \varphi(w) \int_{r_{0} e^{i \theta}}^{\infty(\theta)} \lambda(\zeta) E_{m}(\zeta z) \frac{e_{m}(\zeta w)}{\zeta w} d \zeta d w
$$

for every $\varphi \in \mathcal{O}\left(D_{r}\right)$ and $|z|<\varepsilon<r$, where $\theta \in(-\arg w-\pi /(2 k),-\arg w+$ $\pi /(2 k))$ and here the integration in the inner integral is taken over a ray $\left\{r e^{i \theta}: r \geq r_{0}\right\}$.

Since $\lambda(\zeta)$ is an analytic function for $|\zeta| \geq r_{0}$ and is of polynomial growth at infinity, the left-hand side of (9) is a well-defined analytic function in a complex neighbourhood of the origin.

We show that the definition is independent of the choice of the number $r_{0}$ such that $\lambda(\zeta)$ is analytic for $|\zeta| \geq r_{0}$. To this end we take $r_{1} \leq r_{2}$ such that 
$\lambda(\zeta)$ is analytic for $|\zeta| \geq r_{1}$. Observe that for every fixed $z \in D_{\varepsilon}$ the function

$$
w \mapsto \int_{r_{1} e^{i \theta}}^{r_{2} e^{i \theta}} \lambda(\zeta) E_{m}(\zeta z) \frac{e_{m}(\zeta w)}{\zeta w} d \zeta
$$

is analytic for $w \in D_{r}$ and $\theta \in(-\arg w-\pi /(2 k),-\arg w+\pi /(2 k))$. Hence, by the Cauchy integral formula

$$
\oint_{|w|=\varepsilon} \varphi(w) \int_{r_{1} e^{i \theta}}^{r_{2} e^{i \theta}} \lambda(\zeta) E_{m}(\zeta z) \frac{e_{m}(\zeta w)}{\zeta w} d \zeta d w=0 .
$$

Therefore the right-hand side of (9) is independent of the choice of $r_{0}$.

Hence, in particular the definition of moment pseudodifferential operator $\lambda\left(\partial_{m, z}\right)$, where $\lambda(\zeta)=\zeta^{n}$, coincides with the formula (7) on the moment differential operator $\partial_{m, z}^{n}$.

Definition 9. We define a pole order $q \in \boldsymbol{Q}$ and a leading term $\lambda \in \boldsymbol{C} \backslash\{0\}$ of $\lambda(\zeta)$ as the numbers satisfying the formula

$$
\lim _{\zeta \rightarrow \infty} \frac{\lambda(\zeta)}{\zeta^{q}}=\lambda
$$

which is denoted by $\lambda(\zeta) \sim \lambda \zeta^{q}$.

We have the following estimation

Lemma 1. Let $\varphi \in \mathcal{O}(D)$ and $\lambda\left(\partial_{m, z}\right)$ be a moment pseudodifferential operator with $\lambda(\zeta) \sim \lambda \zeta^{q}$ and $q>0$. Then there exist $r>0$ and $A, B<\infty$ such that

$$
\sup _{|z|<r}\left|\lambda^{j}\left(\partial_{m, z}\right) \varphi(z)\right| \leq|\lambda|^{j} A B^{j q} \Gamma(1+j q / k) \quad \text { for } j=0,1, \ldots
$$

Proof. Since $\lambda(\zeta) \sim \lambda \zeta^{q}$, we may assume that $|\lambda(\zeta)| \leq 2|\lambda||\zeta|^{q}$ for $|\zeta| \geq r_{0}$. Hence, by the definition of kernel functions we have

$$
\begin{aligned}
\left|\int_{r_{0} e^{i \theta}}^{\infty(\theta)} \lambda^{j}(\zeta) E_{m}(\zeta z) \frac{e_{m}(\zeta w)}{\zeta w} d \zeta\right| & \leq \int_{r_{0}}^{\infty} 2^{j}|\lambda|^{j} s^{j q} A_{1} e^{b_{1}|z|^{k} s^{k}} \frac{A_{2} e^{-b_{2}|w|^{k} s^{k}}}{s|w|} d s \\
& \leq \frac{2^{j}|\lambda|^{j} A_{1} A_{2}}{|w|} \int_{0}^{\infty} s^{j q-1} e^{\left(b_{1}|z|^{k}-b_{2}|w|^{k}\right) s^{k}} d s \\
& \stackrel{\sigma=s^{k}}{\leq} \frac{2^{j}|\lambda|^{j} A_{1} A_{2}}{k|w|} \int_{0}^{\infty} \sigma^{j q / k-1} e^{\left(b_{1}|z|^{k}-b_{2}|w|^{k}\right) \sigma} d \sigma \\
& \leq|\lambda|^{j} \tilde{A} \tilde{B}^{j q} \frac{\Gamma(1+j q / k)}{|w|\left(b_{2}|w|^{k}-b_{1}|z|^{k}\right)^{j q / k}} .
\end{aligned}
$$


We choose $r>0$ such that $b_{2} \varepsilon^{k}-b_{1} r^{k}>b_{2} \varepsilon^{k} / 2$. Then for $z \in D_{r}$ we have

$$
\begin{aligned}
\left|\lambda^{j}\left(\partial_{m, z}\right) \varphi(z)\right| & \leq \frac{1}{2 \kappa \pi} \oint_{|w|=\varepsilon}|\varphi(w)||\lambda|^{j} \tilde{A} \tilde{B}^{j q} \frac{\Gamma(1+j q / k)}{|w|\left(b_{2}|w|^{k}-b_{1}|z|^{k}\right)^{j q / k}} d|w| \\
& \leq|\lambda|^{j} \tilde{A} \tilde{B}^{j q} \frac{\Gamma(1+j q / k)}{\varepsilon\left(b_{2} \varepsilon^{k} / 2\right)^{j q / k}} \frac{1}{2 \kappa \pi} \oint_{|w|=\varepsilon}|\varphi(w)| d|w| \\
& \leq|\lambda|^{j} A B^{j q} \Gamma(1+j q / k) .
\end{aligned}
$$

\section{Formal solutions}

In this section we study formal solutions of the initial value problem for a general linear moment partial differential equation with constant coefficients

$$
\left\{\begin{array}{l}
P\left(\partial_{m_{1}, t}, \partial_{m_{2}, z}\right) u=0 \\
\partial_{m_{1}, t}^{j} u(0, z)=\varphi_{j}(z) \in \mathcal{O}(D) \quad \text { for } j=0, \ldots, n-1,
\end{array}\right.
$$

where

$$
P(\lambda, \zeta)=P_{0}(\zeta) \lambda^{n}-\sum_{j=1}^{n} P_{j}(\zeta) \lambda^{n-j}
$$

is a general polynomial of two variables, which is of order $n$ with respect to $\lambda$.

If $P_{0}(\zeta) \neq$ const. then a formal solution of $(10)$ is not uniquely determined.

To avoid this inconvenience we shall choose some special solution which is called the normalised formal solution (see also Balser [3] and Michalik [15]). To this end we factorise the moment differential operator $P\left(\partial_{m_{1}, t}, \partial_{m_{2}, z}\right)$ as follows

$$
\begin{aligned}
P\left(\partial_{m_{1}, t}, \partial_{m_{2}, z}\right) & =P_{0}\left(\partial_{m_{2}, z}\right)\left(\partial_{m_{1}, t}-\lambda_{1}\left(\partial_{m_{2}, z}\right)\right)^{n_{1}} \ldots\left(\partial_{m_{1}, t}-\lambda_{l}\left(\partial_{m_{2}, z}\right)\right)^{n_{l}} \\
& =: P_{0}\left(\partial_{m_{2}, z}\right) \tilde{P}\left(\partial_{m_{1}, t}, \partial_{m_{2}, z}\right)
\end{aligned}
$$

where $\lambda_{1}(\zeta), \ldots, \lambda_{l}(\zeta)$ are the roots of the characteristic equation $P(\lambda, \zeta)=0$ of (10) with multiplicities $n_{1}, \ldots, n_{l}\left(n_{1}+\cdots+n_{l}=n\right)$ respectively.

Since $\lambda_{j}(\zeta)$ is an algebraic function, $\lambda_{j}(\zeta) \sim \lambda_{j} \zeta^{q_{j}}\left(q_{j}=\mu_{j} / v_{j}\right.$ for some relatively prime numbers $\mu_{j} \in \boldsymbol{Z}$ and $\left.v_{j} \in \boldsymbol{N}\right)$ and, moreover, $\lambda_{j}(\zeta)$ is a holomorphic function of the variable $\xi=\zeta^{1 / \kappa}$ for $|\zeta| \geq r_{0}$ (for some $\kappa \in N$ such that $v_{j} \mid \kappa$ and for sufficiently large $\left.r_{0}\right)$. Since $P\left(\partial_{m_{1}, t}, \partial_{m_{2}, z}\right) u=0$ if and only if $P\left(\partial_{m_{1}, t}, \partial_{\tilde{m}_{2}, z}^{k}\right) v=0$ with $v(t, z)=u\left(t, z^{\kappa}\right)$ and $\tilde{m}_{2}(u)=m_{2}(u / \kappa)$ (see Lemma 3), without loss of generality we assume in the paper that $\kappa=1$ and $\lambda_{j}(\zeta)$ is a holomorphic function for $|\zeta| \geq r_{0}$. Hence the moment pseudodifferential operator $\lambda_{j}\left(\partial_{m_{2}, z}\right)$ is well-defined.

Now we are ready to define the uniquely determined normalised solution of $(10)$. 
Definition 10. A formal solution $\hat{u}$ of (10) is called the normalised formal solution if $\hat{u}$ is also a solution of the pseudodifferential equation $\tilde{P}\left(\partial_{m_{1}, t}, \partial_{m_{2}, z}\right) \hat{u}$ $=0$.

Our aim is to study the normalised formal solution of (10). We begin by describing the formal solution of simple moment pseudodifferential equation

$$
\left\{\begin{array}{l}
\left(\partial_{m_{1}, t}-\lambda\left(\partial_{m_{2}, z}\right)\right)^{\beta} u=0 \\
\partial_{m_{1}, t}^{j} u(0, z)=0 \quad(j=0, \ldots, \beta-2) \\
\partial_{m_{1}, t}^{\beta-1} u(0, z)=\lambda^{\beta-1}\left(\partial_{m_{2}, z}\right) \varphi(z) \in \mathcal{O}(D) .
\end{array}\right.
$$

We show that

Lemma 2. The formal power series

$$
\hat{u}(t, z)=m_{1}(0) \sum_{j=\beta-1}^{\infty}\left(\begin{array}{c}
j \\
\beta-1
\end{array}\right) \frac{\lambda^{j}\left(\partial_{m_{2}, z}\right) \varphi(z)}{m_{1}(j)} t^{j}
$$

is a formal solution of (11).

Proof. If $\hat{u}$ is the formal power series given by (12), then

$$
\partial_{m_{1}, t}^{n} \hat{u}(t, z)=m_{1}(0) \sum_{j=\beta-1}^{\infty}\left(\begin{array}{c}
j \\
\beta-1
\end{array}\right) \frac{\lambda^{j}\left(\partial_{m_{2}, z}\right) \varphi(z)}{m_{1}(j-n)} t^{j-n}
$$

for $n=0,1, \ldots, \beta-1$. Hence $\hat{u}$ satisfies the initial conditions $\partial_{m_{1}, t}^{n} \hat{u}(0, z)=0$ for $n=0, \ldots, \beta-2$ and $\partial_{m_{1}, t}^{\beta-1} \hat{u}(0, z)=\lambda^{\beta-1}\left(\partial_{m_{2}, z}\right) \varphi(z)$.

To show that $\hat{u}$ satisfies the equation (11), we observe that

$$
\left(\partial_{m_{1}, t}-\lambda\left(\partial_{m_{2}, z}\right)\right) \sum_{j=0}^{\infty} \frac{\lambda^{j}\left(\partial_{m_{2}, z}\right) \varphi(z)}{m_{1}(j)} t^{j}=0 .
$$

Moreover, if $n \geq 1$ then we have

$$
\begin{aligned}
\left(\partial_{m_{1}, t}\right. & \left.-\lambda\left(\partial_{m_{2}, z}\right)\right) \sum_{j=n}^{\infty}\left(\begin{array}{l}
j \\
n
\end{array}\right) \frac{\lambda^{j}\left(\partial_{m_{2}, z}\right) \varphi(z)}{m_{1}(j)} t^{j} \\
& =\sum_{j=n-1}^{\infty}\left(\begin{array}{c}
j+1 \\
n
\end{array}\right) \frac{\lambda^{j+1}\left(\partial_{m_{2}, z}\right) \varphi(z)}{m_{1}(j)} t^{j}-\sum_{j=n}^{\infty}\left(\begin{array}{l}
j \\
n
\end{array}\right) \frac{\lambda^{j+1}\left(\partial_{m_{2}, z}\right) \varphi(z)}{m_{1}(j)} t^{j} \\
& =\lambda\left(\partial_{m_{2}, z}\right) \sum_{j=n-1}^{\infty}\left(\begin{array}{c}
j \\
n-1
\end{array}\right) \frac{\lambda^{j}\left(\partial_{m_{2}, z}\right) \varphi(z)}{m_{1}(j)} t^{j}
\end{aligned}
$$

since

$$
\left(\begin{array}{c}
j+1 \\
n
\end{array}\right)-\left(\begin{array}{l}
j \\
n
\end{array}\right)=\left(\begin{array}{c}
j \\
n-1
\end{array}\right)
$$


Hence

$$
\begin{aligned}
& \left(\partial_{m_{1}, t}-\lambda\left(\partial_{m_{2}, z}\right)\right)^{\beta} m_{1}(0) \sum_{j=\beta-1}^{\infty}\left(\begin{array}{c}
j \\
\beta-1
\end{array}\right) \frac{\lambda^{j}\left(\partial_{m_{2}, z}\right) \varphi(z)}{m_{1}(j)} t^{j} \\
& =\left(\partial_{m_{1}, t}-\lambda\left(\partial_{m_{2}, z}\right)\right)^{\beta-1} m_{1}(0) \lambda\left(\partial_{m_{2}, z}\right) \sum_{j=\beta-2}^{\infty}\left(\begin{array}{c}
j \\
\beta-2
\end{array}\right) \frac{\lambda^{j}\left(\partial_{m_{2}, z}\right) \varphi(z)}{m_{1}(j)} t^{j} \\
& =\cdots=\left(\partial_{m_{1}, t}-\lambda\left(\partial_{m_{2}, z}\right)\right) m_{1}(0) \lambda^{\beta-1}\left(\partial_{m_{2}, z}\right) \sum_{j=0}^{\infty} \frac{\lambda^{j}\left(\partial_{m_{2}, z}\right) \varphi(z)}{m_{1}(j)} t^{j}=0
\end{aligned}
$$

which shows that $\hat{u}$ given by (12) is a solution of (11).

Next, we have

Proposition 4. If $\hat{u}$ is a formal solution of

$$
\left\{\begin{array}{l}
\tilde{P}\left(\partial_{m_{1}, t}, \partial_{m_{2}, z}\right) \hat{u}=0 \\
\partial_{m_{1}, t}^{j} \hat{u}(0, z)=0 \quad(j=0, \ldots, n-2) \\
\partial_{m_{1}, t}^{n-1} \hat{u}(0, z)=\varphi(z) \in \mathcal{O}(D)
\end{array}\right.
$$

then $\hat{u}=\sum_{\alpha=1}^{l} \sum_{\beta=1}^{n_{\alpha}} \hat{u}_{\alpha \beta}$ with $\hat{u}_{\alpha \beta}$ being a formal solution of

$$
\left\{\begin{array}{l}
\left(\partial_{m_{1}, t}-\lambda_{\alpha}\left(\partial_{m_{2}, z}\right)\right)^{\beta} \hat{u}_{\alpha \beta}=0 \\
\partial_{m_{1}, t}^{j} \hat{u}_{\alpha \beta}(0, z)=0 \quad(j=0, \ldots, \beta-2) \\
\partial_{m_{1}, t}^{\beta-1} \hat{u}_{\alpha \beta}(0, z)=\lambda_{\alpha}^{\beta-1}\left(\partial_{m_{2}, z}\right) \varphi_{\alpha \beta}(z),
\end{array}\right.
$$

where $\varphi_{\alpha \beta}(z):=d_{\alpha \beta}\left(\partial_{m_{2}, z}\right) \varphi(z) \in \mathcal{O}(D)$ and $d_{\alpha \beta}(\zeta)$ is some holomorphic function of polynomial growth.

Proof. Observe that the formal solution $\hat{u}$ of (14) is given by

$$
\hat{u}(t, z)=m_{1}(0) \sum_{j=0}^{\infty} q_{j}\left(\partial_{m_{2}, z}\right) \varphi(z) \frac{t^{j}}{m_{1}(j)},
$$

where $q_{j}(\zeta)$ are the solutions of the difference equation

$$
P_{0}(\zeta) q_{j}(\zeta)=\sum_{k=1}^{n} P_{k}(\zeta) q_{j-k}(\zeta) \quad \text { for } j \geq n
$$

with the initial conditions $q_{0}(\zeta)=q_{1}(\zeta)=\cdots=q_{n-2}(\zeta)=0$ and $q_{n-1}(\zeta)=1$. Since the solutions $q_{j}(\zeta)$ are rational functions, it follows that the moment pseudodifferential operators $q_{j}\left(\partial_{m_{2}, z}\right)$ are well-defined. Moreover, according to 
the theory of difference equations, we have

$$
q_{j}(\zeta)=\sum_{\alpha=1}^{l} \sum_{\beta=1}^{\min \left\{j+1, n_{\alpha}\right\}} c_{\alpha \beta}(\zeta) \frac{j !}{(j+1-\beta) !} \lambda_{\alpha}^{j}(\zeta),
$$

where $c_{\alpha \beta}(\zeta)$ is a holomorphic function of polynomial growth for sufficiently large $|\zeta|$ (say, for $|\zeta| \geq r_{0}$ ) and $\lambda_{\alpha}(\zeta)$ is the root of the characteristic equation with multiplicity $n_{\alpha}$. Combining (16) and (17) we obtain

$$
\hat{u}(t, z)=m_{1}(0) \sum_{\alpha=1}^{l} \sum_{\beta=1}^{n_{\alpha}} c_{\alpha \beta}\left(\partial_{m_{2}, z}\right) \sum_{j=\beta-1}^{\infty} \frac{j !}{(j+1-\beta) !} \lambda_{\alpha}^{j}\left(\partial_{m_{2}, z}\right) \varphi(z) \frac{t^{j}}{m_{1}(j)} .
$$

It means that $\hat{u}=\sum_{\alpha=1}^{l} \sum_{\beta=1}^{n_{\alpha}} \hat{u}_{\alpha \beta}$, where

$$
\hat{u}_{\alpha \beta}(t, z)=m_{1}(0) c_{\alpha \beta}\left(\partial_{m_{2}, z}\right) \sum_{j=\beta-1}^{\infty} \frac{j !}{(j+1-\beta) !} \lambda_{\alpha}^{j}\left(\partial_{m_{2}, z}\right) \varphi(z) \frac{t^{j}}{m_{1}(j)} .
$$

By Lemma 2, the formal power series $\hat{u}_{\alpha \beta}$ is a solution of (15) with $d_{\alpha \beta}(\zeta)=$ $(\beta-1) ! c_{\alpha \beta}(\zeta)$.

We generalise the above result as follows.

Theorem 1. If $\hat{u}$ is the normalised formal solution of (10) then $\hat{u}=$ $\sum_{\alpha=1}^{l} \sum_{\beta=1}^{n_{\alpha}} \hat{u}_{\alpha \beta}$ with $\hat{u}_{\alpha \beta}$ being a formal solution of

$$
\left\{\begin{array}{l}
\left(\partial_{m_{1}, t}-\lambda_{\alpha}\left(\partial_{m_{2}, z}\right)\right)^{\beta} \hat{u}_{\alpha \beta}=0 \\
\partial_{m_{1}, t}^{j} \hat{u}_{\alpha \beta}(0, z)=0 \quad(j=0, \ldots, \beta-2) \\
\partial_{m_{1}, t}^{\beta-1} \hat{u}_{\alpha \beta}(0, z)=\lambda_{\alpha}^{\beta-1}\left(\partial_{m_{2}, z}\right) \varphi_{\alpha \beta}(z),
\end{array}\right.
$$

where $\varphi_{\alpha \beta}(z):=\sum_{j=0}^{n-1} d_{\alpha \beta j}\left(\partial_{m_{2}, z}\right) \varphi_{j}(z) \in \mathcal{O}(D)$ for some holomorphic functions $d_{\alpha \beta j}(\zeta)$ of polynomial growth.

Proof. Applying the principle of superposition of solutions of linear equation in the same way as in $[15$, Remark 2] and repeating the proof of Proposition 4, we obtain the assertion.

The next lemma allows us to change the order of equation. It is especially useful in the case when the moment function $m(u)$ is of order $1 / k \geq 2$.

Lemma 3. Let $m_{1}(u)$ and $m_{2}(u)$ be moment functions of orders $1 / k_{1}>0$ and $1 / k_{2}>0$ respectively and $\mu, v \in N$. Then $\tilde{m}_{1}(u):=m_{1}(u / \mu)$ and $\tilde{m}_{2}(u):=$ $m_{2}(u / v)$ are moment functions of order $1 / \mu k_{1}$ and $1 / v k_{2}$ respectively. Moreover, $\hat{u}=\hat{u}(t, z)$ is a formal solution of

$$
\left\{\begin{array}{l}
P\left(\partial_{m_{1}, t}, \partial_{m_{2}, z}\right) \hat{u}=0 \\
\partial_{m_{1}, t}^{k} \hat{u}(0, z)=\varphi_{k}(z), \quad k=0, \ldots, n-1 .
\end{array}\right.
$$


if and only if $\hat{v}(t, z):=\hat{u}\left(t^{\mu}, z^{v}\right)$ is a formal solution of

$$
\left\{\begin{array}{l}
P\left(\partial_{\tilde{m}_{1}, t}^{\mu}, \partial_{\tilde{m}_{2}, z}^{v}\right) \hat{v}=0, \\
\partial_{\tilde{m}_{1}, t}^{j} \hat{v}(0, z)=\varphi_{k}\left(z^{v}\right) \quad \text { for } j=k \mu, k=0, \ldots, n-1, \\
\partial_{\tilde{m}_{1}, t}^{j} \hat{v}(0, z)=0 \quad \text { for } j=1, \ldots, n \mu-1 \text { and } \mu \nmid j .
\end{array}\right.
$$

Proof. By Definition 6 and Remark 1, we see that $\tilde{m}_{1}(u)=m_{1}(u / \mu)$ and $\tilde{m}_{2}(u)=m_{2}(u / v)$ are moment functions of order $1 / \mu k_{1}$ and $1 / v k_{2}$ respectively.

To prove the second part of the lemma, we assume that $\hat{u}(t, z)=$ $m_{1}(0) \sum_{n=0}^{\infty} u_{n}(z) t^{n} / m_{1}(n)$. Then

$$
\begin{aligned}
\left(\partial_{m_{1}, t} \hat{u}\right)\left(t^{\mu}, z^{v}\right) & =m_{1}(0) \sum_{n=0}^{\infty} \frac{u_{n+1}\left(z^{v}\right)}{m_{1}(n)} t^{\mu n}=\tilde{m}_{1}(0) \sum_{n=0}^{\infty} \frac{u_{n+1}\left(z^{v}\right)}{\tilde{m}_{1}(\mu n)} t^{\mu n} \\
& =\partial_{\tilde{m}_{1}, t}^{\mu}\left(\hat{u}\left(t^{\mu}, z^{v}\right)\right)
\end{aligned}
$$

and analogously

$$
\left(\partial_{m_{2}, z} \hat{u}\right)\left(t^{\mu}, z^{v}\right)=\partial_{\tilde{m}_{2}, z}^{v}\left(\hat{u}\left(t^{\mu}, z^{v}\right)\right)
$$

Hence

$$
P\left(\partial_{m_{1}, t}, \partial_{m_{2}, z}\right) \hat{u}=0 \quad \text { if and only if } \quad P\left(\partial_{\tilde{m}_{1}, t}^{\mu}, \partial_{\tilde{m}_{2}, z}^{v}\right) \hat{v}=0 .
$$

Moreover $\hat{u}$ satisfies the initial conditions in (18) if and only if $\hat{v}$ satisfies the initial conditions in (19), which proves the assertion.

\section{Gevrey estimates}

In this section we study the Gevrey order of formal solution $\hat{u}$ of (10), which depends on the orders $1 / k_{1}, 1 / k_{2}$ of moment functions $m_{1}(u), m_{2}(u)$ respectively, and depends on the pole orders $q_{\alpha}$ of the roots $\lambda_{\alpha}(\zeta)(\alpha=1, \ldots, l)$ of the characteristic equation. First, we consider the simple moment pseudodifferential equation (11). We have

Proposition 5. We assume that $\hat{u}$ is a formal solution of (11) and $q>0$ is a pole order of $\lambda(\zeta)$. Then we have

(i) If $1 / k_{1}<q / k_{2}$ then $\hat{u}$ is a Gevrey series of order $q / k_{2}-1 / k_{1}$ with respect to $t$.

(ii) If $1 / k_{1}=q / k_{2}$ then $u \in \mathcal{O}\left(D^{2}\right)$.

(iii) If $1 / k_{1}>q / k_{2}$ then $u \in \mathcal{O}^{k_{1} k_{2} /\left(k_{2}-q k_{1}\right)}(C \times D)$.

Proof. We estimate the coefficients of the formal solution $\hat{u}(t, z)=$ $\sum_{j=0}^{\infty} u_{j}(z) t^{j}$ of (11). By Lemmas 1 and 2 we have

$$
\left|u_{j}(z)\right|=m_{1}(0)\left(\begin{array}{c}
j \\
\beta-1
\end{array}\right) \frac{\left|\lambda^{j}\left(\partial_{m_{2}, z}\right) \varphi(z)\right|}{m_{1}(j)} \leq A B^{j} \frac{\Gamma\left(1+j q / k_{2}\right)}{\Gamma\left(1+j / k_{1}\right)} \quad \text { for } z \in D .
$$


Hence $\hat{u}$ is a Gevrey series of order $q / k_{2}-1 / k_{1}$ for $1 / k_{1}<q / k_{2}$, a convergent series in a complex neighbourhood of the origin for $1 / k_{1}=q / k_{2}$ and an entire function for $1 / k_{1}>q / k_{2}$. In the last case, by the properties of the MittagLeffler function (see [2, p. 234]), we have

$$
\begin{aligned}
|u(t, z)| & \leq \sum_{j=0}^{\infty} A B^{j} \frac{\Gamma\left(1+j q / k_{2}\right)}{\Gamma\left(1+j / k_{1}\right)}|t|^{j} \leq \sum_{j=0}^{\infty} A B^{j} \frac{|t|^{j}}{\Gamma\left(1+j\left(1 / k_{1}-q / k_{2}\right)\right)} \\
& \leq A \boldsymbol{E}_{1 / k_{1}-q / k_{2}}(B|t|) \leq \tilde{A} e^{\tilde{B}|t|^{k_{1} k_{2} /\left(k_{2}-q k_{1}\right)}}
\end{aligned}
$$

where $\boldsymbol{E}_{1 / k_{1}-q / k_{2}}$ is the Mittag-Leffler function of index $1 / k_{1}-q / k_{2}$.

Combining Theorem 1 and Proposition 5 we obtain

Theorem 2. Let $\hat{u}$ be the normalised formal solution of (10) with the decomposition $\hat{u}=\sum_{\alpha=1}^{l} \sum_{\beta=1}^{n_{\alpha}} \hat{u}_{\alpha \beta}$ constructed in Theorem 1 and let $q_{\alpha}>0$ be a pole order of $\lambda_{\alpha}(\zeta)$. Then a formal series $\hat{u}_{\alpha \beta}$ is characterised as follows:

- If $1 / k_{1}<q_{\alpha} / k_{2}$ then $\hat{u}_{\alpha \beta}$ is a Gevrey series of order $q_{\alpha} / k_{2}-1 / k_{1}$ with respect to $t$.

- If $1 / k_{1}=q_{\alpha} / k_{2}$ then $u_{\alpha \beta} \in \mathcal{O}\left(D^{2}\right)$.

- If $1 / k_{1}>q_{\alpha} / k_{2}$ then $u_{\alpha \beta} \in \mathcal{O}^{k_{1} k_{2} /\left(k_{2}-q_{\alpha} k_{1}\right)}(\boldsymbol{C} \times D)$.

\section{Analytic solutions}

In this section we study the convergent solutions $u$ of (11). Therefore by Proposition 5 we assume that $1 / k_{1} \geq q / k_{2}$, where $1 / k_{1}, 1 / k_{2}$ are orders of moment functions $m_{1}(u), m_{2}(u)$ respectively, and $q=\mu$ is a pole order of $\lambda(\zeta)$ for $\mu \in N$. Moreover, we assume that $\lambda(\zeta)$ is a holomorphic function for $|\zeta| \geq r_{0}$ (for some $r_{0}>0$ ). We find a characterisation of analytic continuation property of $u$ in terms of the Cauchy data $\varphi$.

First, we introduce the following integral representation of solution.

Lemma 4. Let $u$ be a solution of (11) and $1 / k_{1} \geq q / k_{2}$. Then $u$ is analytic in some complex neighbourhood of the origin and is given by

$$
u(t, z)=\frac{t^{\beta-1}}{(\beta-1) !} \partial_{t}^{\beta-1} \frac{m_{1}(0)}{2 \kappa \pi i} \oint_{|w|=\varepsilon} \varphi(w) \int_{r_{0} e^{i \theta}}^{\infty(\theta)} E_{m_{1}}(t \lambda(\zeta)) E_{m_{2}}(\zeta z) \frac{e_{m_{2}}(\zeta w)}{\zeta w} d \zeta d w
$$

with $\theta \in\left(-\arg w-\pi /\left(2 k_{2}\right),-\arg w+\pi /\left(2 k_{2}\right)\right)$.

Proof. Since $1 / k_{1} \geq q / k_{2}$, by Proposition 5 the solution $u$ is analytic in some complex neighbourhood of the origin. To show that the inner integral on the right-hand side of (20) is convergent, observe that by Definitions 5 and 6 there exist constants $A_{i}$ and $b_{i}(i=1,2,3)$ such that $\left|E_{m_{1}}(t \lambda(\zeta))\right| \leq A_{1} e^{b_{1}|t|^{k_{1}}|\zeta|^{k_{1} q}}$, 
$\left|E_{m_{2}}(\zeta z)\right| \leq A_{2} e^{b_{2}|\zeta|^{k_{2}}|z|^{k_{2}}}$ and $\left|e_{m_{2}}(\zeta w)\right| \leq A_{3} e^{-b_{3}|\zeta|^{k_{2}}|w|^{k_{2}}}$. Hence, for fixed $w \in$ $C \backslash\{0\}$ such that $|z|$ is small relative to $|w|$ and for $|t|<a|w|^{q}$ with some fixed $a>0$, we have

$$
\left|\int_{r_{0} e^{i \theta}}^{\infty(\theta)} E_{m_{1}}(t \lambda(\zeta)) E_{m_{2}}(\zeta z) \frac{e_{m_{2}}(\zeta w)}{\zeta w} d \zeta\right| \leq \int_{r_{0}}^{\infty} \tilde{A} e^{-\tilde{b} s^{k_{2}}|w|^{k_{2}}} d s<\infty .
$$

It means that the right-hand side of (20) is a well-defined holomorphic function in some complex neighbourhood of the origin. To show (20), observe that by Lemma 2 and by (9) we have

$$
\begin{aligned}
u(t, z) & =m_{1}(0) \sum_{j=\beta-1}^{\infty}\left(\begin{array}{c}
j \\
\beta-1
\end{array}\right) \frac{\lambda^{j}\left(\partial_{m_{2}, z}\right) \varphi(z)}{m_{1}(j)} t^{j} \\
& =\frac{m_{1}(0) t^{\beta-1}}{(\beta-1) !} \partial_{t}^{\beta-1} \sum_{j=0}^{\infty} \frac{\lambda^{j}\left(\partial_{m_{2}, z}\right) \varphi(z)}{m_{1}(j)} t^{j} \\
& =\frac{t^{\beta-1}}{(\beta-1) !} \partial_{t}^{\beta-1} \sum_{j=0}^{\infty} \frac{t^{j}}{m_{1}(j)} \frac{m_{1}(0)}{2 \kappa \pi i} \oint_{|w|=\varepsilon} \varphi(w) \int_{r_{0} e^{i \theta}}^{\infty(\theta)} \lambda^{j}(\zeta) E_{m_{2}}(\zeta z) \frac{e_{m_{2}}(\zeta w)}{\zeta w} d \zeta d w \\
& =\frac{t^{\beta-1}}{(\beta-1) !} \partial_{t}^{\beta-1} \frac{m_{1}(0)}{2 \kappa \pi i} \oint_{|w|=\varepsilon} \varphi(w) \int_{r_{0} e^{i \theta}}^{\infty(\theta)} \sum_{j=0}^{\infty} \frac{t^{j} \lambda^{j}(\zeta)}{m_{1}(j)} E_{m_{2}}(\zeta z) \frac{e_{m_{2}}(\zeta w)}{\zeta w} d \zeta d w .
\end{aligned}
$$

In the next crucial lemma we use the integral representation (20) of solution $u$ to find its analytic continuation.

Lemma 5. Let $\lambda(\zeta) \sim \lambda \zeta^{q}$ for $q=\mu$ with $\mu \in N$, where $\lambda(\zeta)$ is an analytic function for $|\zeta| \geq r_{0}$ (for some $\left.r_{0}>0\right)$. Moreover, let $1 / k_{1}=q / k_{2}, K>0$ and $d \in \boldsymbol{R}$. We assume that $u$ is a solution of

$$
\left\{\begin{array}{l}
\left(\partial_{m_{1}, t}-\lambda\left(\partial_{m_{2}, z}\right)\right)^{\beta} u=0 \\
\partial_{m_{1}, t}^{j} u(0, z)=\varphi_{j}(z) \in \mathcal{O}(D) \quad(j=0, \ldots, \beta-1) .
\end{array}\right.
$$

If $\varphi_{j} \in \mathcal{O}^{q K}\left(\hat{S}_{(d+\arg \lambda+2 k \pi) / q}\right)$ for $k=0, \ldots, \mu-1$ and $j=0, \ldots, \beta-1$, then $u \in$ ${ }^{K}\left(\hat{S}_{d} \times D\right)$.

Proof. First, we consider case $k_{1}>1 / 2$. By the principle of superposition of solutions of linear equations we may assume that $u$ satisfies (11) with $\varphi \in$ $\mathcal{O}^{q K}\left(\hat{S}_{(d+\arg \lambda+2 k \pi) / q}(\tilde{\delta})\right)$ for $k=0, \ldots, \mu-1$ and for some $\tilde{\delta}>0$. So, by Lemma $4, u$ is given by (20). Next, observe that the function

$$
t \mapsto \int_{r_{0} e^{i \theta}}^{\infty(\theta)} E_{m_{1}}(t \lambda(\zeta)) E_{m_{2}}(\zeta z) \frac{e_{m_{2}}(\zeta w)}{\zeta w} d \zeta
$$


which is holomorphic on $\left\{t \in \boldsymbol{C}:|t|<a|w|^{q}\right\}$ for every fixed $w \in C \backslash\{0\}$, can be also analytically continued to the set

$$
\{t \in \tilde{\boldsymbol{C}}:(\arg t+2 k \pi+\arg \lambda) / \mu \neq \arg w+2 n \pi \text { for every } k, n \in \boldsymbol{Z}\} .
$$

Indeed, we may replace a direction $\theta$ in (22) by $\tilde{\theta}$ satisfying the following conditions

- $\arg t+2 k \pi+\arg \lambda+q \tilde{\theta} \in\left(\pi /\left(2 k_{1}\right), 2 \pi-\pi /\left(2 k_{1}\right)\right)$ for some $k \in \boldsymbol{Z}$ (in this case, by Definition 5 , we have $\left|E_{m_{1}}(t \lambda(\zeta))\right| \leq C|t \lambda(\zeta)|^{-1}$ as $\zeta \rightarrow \infty$, $\arg \zeta=\tilde{\theta})$,

- $\arg w+2 n \pi+\tilde{\theta} \in\left(-\pi /\left(2 k_{2}\right), \pi /\left(2 k_{2}\right)\right)$ for some $n \in \boldsymbol{Z}$ (in this case, by Definitions 5 and 6 , there exists $\varepsilon>0$ such that $\left|E_{m_{2}}(\zeta z) e_{m_{2}}(\zeta w) /(\zeta w)\right| \leq e^{-\varepsilon|\zeta|^{k_{2}}}$ as $\zeta \rightarrow \infty, \arg \zeta=\tilde{\theta})$.

Since $q=k_{2} / k_{1}=\mu$, these requirements may be together satisfied under the condition that $(\arg t+2 k \pi+\arg \lambda) / \mu \neq \arg w+2 n \pi$ for every $k, n \in Z$. Therefore the function (22) is analytically continued to the sectors (23) and is bounded as $t \rightarrow \infty$.

To estimate $u$, fix $z$ such close to the origin, that $\arg (w-z) \approx \arg w$ along a circle $|w|=\varepsilon$. Repeating the proof of Theorem 3.1 in [7], we split this circle into $2 \mu$ arcs $\gamma_{2 k}$ and $\gamma_{2 k+1}(k=0, \ldots, \mu-1)$, where $\gamma_{2 k}$ extends between points of argument $(d+\arg \lambda+2 k \pi) / q \pm \tilde{\delta} / 3$ and $\gamma_{2 k+1}$ extends between $(d+\arg \lambda+$ $2 k \pi) / q+\tilde{\delta} / 3$ and $(d+\arg \lambda+2(k+1) \pi) / q-\tilde{\delta} / 3 \bmod 2 \pi$. Finally, since $\varphi \in$ $\mathcal{O}\left(S_{(d+\arg \lambda+2 k \pi) / q}(\tilde{\delta})\right)$, we may deform $\gamma_{2 k}$ into a path $\gamma_{2 k}^{R}$ along the ray arg $w=$ $(d+\arg \lambda+2 k \pi) / q-\tilde{\delta} / 3$ to a point with modulus $R$ (which can be chosen arbitrarily large), then along the circle $|w|=R$ to the ray $\arg w=(d+\arg \lambda+$ $2 k \pi) / q+\tilde{\delta} / 3$ and back along this ray to the original circle. So, we have

$$
u(t, z)=\frac{t^{\beta-1}}{(\beta-1) !} \partial_{t}^{\beta-1} u_{1}(t, z)+\frac{t^{\beta-1}}{(\beta-1) !} \partial_{t}^{\beta-1} u_{2}(t, z),
$$

where

$$
u_{1}(t, z):=\sum_{k=0}^{\mu-1} \frac{m_{1}(0)}{2 \kappa \pi i} \oint_{\gamma_{2 k}^{R}} \varphi(w) \int_{r_{0} e^{i \theta}}^{\infty(\theta)} E_{m_{1}}(t \lambda(\zeta)) E_{m_{2}}(\zeta z) \frac{e_{m_{2}}(\zeta w)}{\zeta w} d \zeta d w
$$

and

$$
u_{2}(t, z):=\sum_{k=0}^{\mu-1} \frac{m_{1}(0)}{2 \kappa \pi i} \oint_{\gamma_{2 k+1}} \varphi(w) \int_{r_{0} e^{i \theta}}^{\infty(\theta)} E_{m_{1}}(t \lambda(\zeta)) E_{m_{2}}(\zeta z) \frac{e_{m_{2}}(\zeta w)}{\zeta w} d \zeta d w
$$

Note that $R$ may be chosen arbitrarily large and the function (22) is analytic on $\left\{t \in C:|t|\left\langle a|w|^{q}\right\}\right.$ (we assume that $|z|$ is small relative to $|w|$ ). Hence, one 
can find $\delta>0$ such that $u_{1}$ is analytically continued to $S_{d}(\delta) \times D$. Estimating this integral we have

$$
\max _{z \in D}\left|u_{1}(t, z)\right| \leq \frac{\mu}{2 \kappa \pi} \max _{k=0, \ldots, \mu-1} \oint_{\gamma_{2 k}^{R}}|\varphi(w)| C d|w| \leq A R e^{B R^{q K}} \leq \tilde{A} e^{\tilde{B}|t|^{K}}
$$

since $|t| \sim|w|^{q}=R^{q}$.

Moreover, since the function (22) is analytically continued into the region (23), we see that $u_{2}$ is also analytically continued to $S_{d+2 n \pi / v}(\delta) \times D$ for $n=$ $0, \ldots, v-1$. Estimating in a similar way to (24), we conclude that $u_{2}$ is bounded as $t \rightarrow \infty$.

Hence also $u$ is analytically continued to $S_{d+2 n \pi / v}(\delta) \times D$ for $n=0, \ldots$, $v-1$ and is of exponential growth of order at most $K$ as $t \rightarrow \infty$.

In case $k_{1} \leq 1 / 2$ there exists $p \in N$ such that $\tilde{k}_{1}:=p k_{1}>1 / 2$. By Lemma $3, v(t, z):=u\left(t^{p}, z^{p}\right)$ is a solution of

$$
\left\{\begin{array}{l}
\left(\partial_{\tilde{m}_{1}, t}^{p}-\lambda\left(\partial_{\tilde{m}_{2}, z}^{p}\right)\right)^{\beta} v=0 \\
\partial_{\tilde{m}_{1}, t}^{n p} v(0, z)=\varphi_{n}\left(z^{p}\right) \in \mathcal{O}^{q K}\left(\hat{S}_{(d+\arg \lambda+2 k \pi) /(p q)}\right) \text { for } n=0, \ldots, \beta-1 \\
\partial_{\tilde{m}_{1}, t}^{j} v(0, z)=0 \text { for } j=1, \ldots, \beta p-1 \text { and } p \nmid j
\end{array}\right.
$$

where $\tilde{m}_{1}(u)=m_{1}(u / p)$ is a moment function of order $1 / \tilde{k}_{1}$. satisfy

By Theorem 1 we have $v=v_{0}+\cdots+v_{p-1}$, where $v_{j}(j=0, \ldots, p-1)$

$$
\left\{\begin{array}{l}
\left(\partial_{\tilde{m}_{1}, t}-e^{i 2 j \pi / p} \lambda^{1 / p}\left(\partial_{\tilde{m}_{2}, z}^{p}\right)\right)^{\beta} v_{j}=0, \\
\partial_{\tilde{m}_{1}, t}^{n} v_{j}(0, z)=\tilde{\varphi}_{j n}(z) \in \mathcal{O}^{q K}\left(\hat{S}_{(d+\arg \lambda) / q+2 k \pi / \mu}\right) \text { for } n=0, \ldots, \beta-1 .
\end{array}\right.
$$

Applying the first part of the proof to the above equation we obtain $v_{j}(t, z) \in$ $\mathcal{O}^{p K}\left(\hat{S}_{(d+2 j \pi) / p} \times D\right)$ for $j=1, \ldots, p$. It means that $u(t, z)=v\left(t^{1 / p}, z^{1 / p}\right) \in$ $\mathcal{O}^{K}\left(\hat{S}_{d} \times D\right)$.

To show that the sufficient condition for analytic continuation of $u$ given in Lemma 5 is also necessary, we need the following auxiliary lemmas.

Lemma 6. We assume that $q=1$ and $1 / k_{1}=1 / k_{2}$. Then $u \in \mathcal{O}\left(D^{2}\right)$ satisfies the equation

$$
\left(\partial_{m_{1}, t}-\lambda\left(\partial_{m_{2}, z}\right)\right) u=0
$$

if and only if $u$ is a solution of the equation

$$
\left(\partial_{m_{2}, z}-\lambda^{-1}\left(\partial_{m_{1}, t}\right)\right) u=0 .
$$

Proof. Since the equations (25) and (26) are symmetric, we only need to prove the implication one way. To this end, we assume that $u$ satisfies (25). It 
means, by Lemma 4, that

$$
u(t, z)=\frac{m_{1}(0)}{2 \kappa \pi i} \oint_{|w|=\varepsilon} \varphi(w) \int_{r_{0} e^{i \theta}}^{\infty(\theta)} E_{m_{1}}(t \lambda(\zeta)) E_{m_{2}}(\zeta z) \frac{e_{m_{2}}(\zeta w)}{\zeta w} d \zeta d w
$$

for some $\varphi \in \mathcal{O}(D)$. Hence, by the definition of the pseudodifferential operator $\lambda^{-1}\left(\partial_{m_{1}, t}\right)$ we have

$$
\begin{aligned}
& \lambda^{-1}\left(\partial_{m_{1}, t}\right) u(t, z) \\
& \quad=\frac{m_{1}(0)}{2 \kappa \pi i} \oint_{|w|=\varepsilon} \varphi(w) \int_{r_{0} e^{i \theta}}^{\infty(\theta)} \lambda^{-1}(\lambda(\zeta)) E_{m_{1}}(t \lambda(\zeta)) E_{m_{2}}(\zeta z) \frac{e_{m_{2}}(\zeta w)}{\zeta w} d \zeta d w \\
& \quad=\frac{m_{1}(0)}{2 \kappa \pi i} \oint_{|w|=\varepsilon} \varphi(w) \int_{r_{0} e^{i \theta}}^{\infty(\theta)} E_{m_{1}}(t \lambda(\zeta)) \zeta E_{m_{2}}(\zeta z) \frac{e_{m_{2}}(\zeta w)}{\zeta w} d \zeta d w \\
& \quad=\partial_{m_{2}, z} u(t, z) .
\end{aligned}
$$

So $u$ satisfies also (26).

Repeating the proof of Lemma 6 in [16], we generalise the last result as follows.

Lemma 7. We assume that $q=1$ and $1 / k_{1}=1 / k_{2}$. Then $u \in \mathcal{O}\left(D^{2}\right)$ satisfies the equation

$$
\left(\partial_{m_{1}, t}-\lambda\left(\partial_{m_{2}, z}\right)\right)^{\beta} u=0
$$

if and only if $u$ satisfies the equation

$$
\left(\partial_{m_{2}, z}-\lambda^{-1}\left(\partial_{m_{1}, t}\right)\right)^{\beta} u=0 .
$$

Now we can state the main result of the paper.

Theorem 3. Let $\lambda(\zeta)$ be a root of the characteristic equation of (10) and $\lambda(\zeta) \sim \lambda \zeta^{q}$ for $q=\mu$ with $\mu \in N$. Moreover, let us assume that $u$ is a solution of (11), $1 / k_{1}=q / k_{2}, K>0$ and $d \in \boldsymbol{R}$. Then the following conditions are equivalent:

(i) $u \in \mathcal{O}^{K}\left(\hat{S}_{d} \times D\right)$,

(ii) $\varphi \in \mathcal{O}^{q K}\left(\hat{S}_{(d+\arg \lambda+2 k \pi) / q}\right)$ for $k=0, \ldots, \mu-1$.

Proof. To prove the implication (i) $\Rightarrow$ (ii), observe that by Lemma 3 , the function $v(t, z):=u\left(t^{\mu}, z\right)$ satisfies

$$
\left(\partial_{\tilde{m}_{1}, t}^{\mu}-\lambda\left(\partial_{m_{2}, z}\right)\right)^{\beta} v=0,
$$

where $\tilde{m}_{1}(u):=m_{1}(u / \mu)$. It means that $v$ is also a solution of the equation

$$
\left(\partial_{\tilde{m}_{1}, t}-\tilde{\lambda}_{0}\left(\partial_{m_{2}, z}\right)\right)^{\beta} \ldots\left(\partial_{\tilde{m}_{1}, t}-\tilde{\lambda}_{\mu-1}\left(\partial_{m_{2}, z}\right)\right)^{\beta} v=0,
$$


where

$$
\tilde{\lambda}_{j}(\zeta):=e^{i 2 \pi j / \mu} \lambda^{1 / \mu}(\zeta) \quad \text { for } j=0, \ldots, \mu-1 .
$$

Since $\tilde{\lambda}_{j}(\zeta) \sim e^{i 2 \pi j / \mu} \lambda^{1 / \mu \zeta}$, by Lemma 7 and by the condition (i), $v$ satisfies also

$$
\left\{\begin{array}{l}
\left(\partial_{m_{2}, z}-\tilde{\lambda}_{0}^{-1}\left(\partial_{\tilde{m}_{1}, t}\right)\right)^{\beta} \ldots\left(\partial_{m_{2}, z}-\tilde{\lambda}_{\mu-1}^{-1}\left(\partial_{\tilde{m}_{1}, t}\right)\right)^{\beta} v=0 \\
\partial_{m_{2}, z}^{n} v(t, 0)=\tilde{\psi}_{n}(t) \in \mathcal{O}^{\mu K}\left(\hat{S}_{(d+2 \pi k) / \mu}\right) \quad \text { for } n=0, \ldots, \mu \beta-1, k=0, \ldots, \mu-1 .
\end{array}\right.
$$

Hence, by Theorem 1, $v=v_{0}+\cdots+v_{\mu-1}$ with $v_{j}(j=0, \ldots, \mu-1)$ satisfying

$$
\left\{\begin{array}{l}
\left(\partial_{m_{2}, z}-\tilde{\lambda}_{j}^{-1}\left(\partial_{\tilde{m}_{1}, t}\right)\right)^{\beta} v_{j}=0, \\
\partial_{m_{2}, z}^{n} v_{j}(t, 0)=\tilde{\psi}_{j n}(t) \in \mathcal{O}^{\mu K}\left(\hat{S}_{(d+2 \pi k) / \mu}\right) \text { for } n=0, \ldots, \beta-1, k=0, \ldots, \mu-1 .
\end{array}\right.
$$

Since $\tilde{\lambda}_{j}^{-1}(\tau) \sim e^{-i 2 \pi j / \mu} \lambda^{-1 / \mu} \tau$, by Lemma 5 with replaced variables, we conclude that $v_{j}(t, z) \in \mathcal{O}^{\mu K}\left(D \times \hat{S}_{\theta_{j k}}\right)$, where

$$
\theta_{j k}:=(d+2 \pi k) / \mu-\arg \left(e^{-i 2 \pi j / \mu} \lambda^{-1 / \mu}\right)=(d+\arg \lambda+2 \pi(k+j)) / \mu
$$

for $k=0, \ldots, \mu-1$. In consequence, also $v(t, z) \in \mathcal{O}^{\mu K}\left(D \times \hat{S}_{(d+\arg \lambda+2 \pi k) / \mu}\right)$ and finally $u(t, z)=v\left(t^{1 / \mu}, z\right) \in \mathcal{O}^{q K}\left(D \times \hat{S}_{(d+\arg \lambda+2 \pi k) / q}\right)$ for $k=0, \ldots, \mu-1$. In particular $\varphi(z) \in \mathcal{O}^{q K}\left(\hat{S}_{(d+\arg \lambda+2 \pi k) / q}\right)$, which proves the implication (i) $\Rightarrow$ (ii).

The implication (ii) $\Rightarrow$ (i) is given immediately by Lemma 5 .

\section{Summable and multisummable solutions}

In this section we characterise the summable formal solutions $\hat{u}$ of (11) in terms of the Cauchy data $\varphi$. Next, we also give a similar characterisation of multisummable normalised formal solutions of general equation (10).

Theorem 4. Let us assume that $\hat{u}$ is a formal solution of $(11), \lambda(\zeta)$ is a root of the characteristic equation of (10), $\lambda(\zeta) \sim \lambda \zeta^{q}, 1 / k_{1}<q / k_{2}, \quad q=\mu$ with $\mu \in N, K=\left(q / k_{2}-1 / k_{1}\right)^{-1}$ and $d \in \boldsymbol{R}$. Then the following conditions are equivalent:

(i) $\hat{u}$ is $K$-summable in the direction $d$,

(ii) $\varphi \in \mathcal{O}^{q K}\left(\hat{S}_{(d+\arg \lambda+2 k \pi) / q}\right)$ for $k=0, \ldots, \mu-1$.

Proof. First, observe that by Lemma 2 and Proposition 2, $\hat{u}$ is $K$ summable in the direction $d$ if and only if

$$
v(t, z):=m_{1}(0) m(0) \sum_{j=\beta-1}^{\infty}\left(\begin{array}{c}
j \\
\beta-1
\end{array}\right) \frac{\lambda^{j}\left(\partial_{m_{2}, z}\right) \varphi(z)}{m_{1}(j) m(j)} t^{j} \in \mathcal{O}^{K}\left(\hat{S}_{d} \times D\right)
$$

with some moment function $m(u)$ of order $K$. 
Moreover, by Proposition 1 , we see that $\tilde{m}_{1}(u):=m_{1}(u) m(u)$ is a moment function of order $1 / \tilde{k}_{1}:=\left(1 / k_{1}+1 / K\right)=q / k_{2}$. It means, by Lemma 2 , that $v$ is a solution of

$$
\left\{\begin{array}{l}
\left(\partial_{\tilde{m}_{1}, t}-\lambda\left(\partial_{m_{2}, z}\right)\right)^{\beta} v=0 \\
\partial_{\tilde{m}_{1}, t}^{j} v(0, z)=0 \quad(j=0, \ldots, \beta-2) \\
\partial_{\tilde{m}_{1}, t}^{\beta-1} v(0, z)=\lambda^{\beta-1}\left(\partial_{m_{2}, z}\right) \varphi(z) \in \mathcal{O}(D) .
\end{array}\right.
$$

Hence, applying Theorem 3 to $v$, we obtain the assertion.

Now we return to the general equation (10). For convenience we assume that

$$
P(\lambda, \zeta)=P_{0}(\zeta) \tilde{P}(\lambda, \zeta)=P_{0}(\zeta) \prod_{\alpha=1}^{\tilde{n}} \prod_{\beta=1}^{l_{\alpha}}\left(\lambda-\lambda_{\alpha \beta}(\zeta)\right)^{n_{\alpha \beta}}
$$

where $\lambda_{\alpha \beta}(\zeta) \sim \lambda_{\alpha \beta} \zeta^{q_{\alpha}}$ is the root of the characteristic equation with $q_{\alpha} \in \boldsymbol{Z}_{+}$ and $\lambda_{\alpha \beta} \in C \backslash\{0\}$ for $\alpha=1, \ldots, \tilde{n}$ and $\beta=1, \ldots, l_{\alpha}$. Without loss of generality we may assume that there exist exactly $N$ pole orders of the roots of the characteristic equation, which are greater than $k_{2} / k_{1}$, say $k_{2} / k_{1}<q_{1}<\cdots<$ $q_{N}<\infty$ and let $K_{\alpha}>0$ be defined by $K_{\alpha}:=\left(q_{\alpha} / k_{2}-1 / k_{1}\right)^{-1}$ for $\alpha=$ $1, \ldots, N$.

By Theorem 1, the normalised formal solution $\hat{u}$ of $(10)$ is given by

$$
\hat{u}=\sum_{\alpha=1}^{\tilde{n}} \sum_{\beta=1}^{l_{\alpha}} \sum_{\gamma=1}^{n_{\alpha \beta}} \hat{u}_{\alpha \beta \gamma}
$$

with $\hat{u}_{\alpha \beta \gamma}$ satisfying

$$
\left\{\begin{array}{l}
\left(\partial_{m_{1}, t}-\lambda_{\alpha \beta}\left(\partial_{m_{2}, z}\right)\right)^{\gamma} \hat{u}_{\alpha \beta \gamma}=0 \\
\partial_{m_{1}, t}^{j} \hat{u}_{\alpha \beta \gamma}(0, z)=0 \text { for } j=0, \ldots, \gamma-2 \\
\partial_{m_{1}, t}^{\gamma-1} \hat{u}_{\alpha \beta \gamma}=\lambda_{\alpha \beta}^{\gamma-1}\left(\partial_{m_{2}, z}\right) \varphi_{\alpha \beta \gamma}(z),
\end{array}\right.
$$

where $\varphi_{\alpha \beta \gamma}(z)=\sum_{j=0}^{n-1} d_{\alpha \beta \gamma j}\left(\partial_{m_{2}, z}\right) \varphi_{j}(z) \in \mathcal{O}(D)$ and $d_{\alpha \beta \gamma j}(\zeta)$ are holomorphic functions of polynomial growth at infinity.

Under the above conditions, immediately by Theorem 4 we have

Theorem 5. Let $\left(d_{1}, \ldots, d_{N}\right) \in \boldsymbol{R}^{N}$ be an admissible multidirection with respect to $\left(K_{1}, \ldots, K_{N}\right)$ and let $q_{\alpha}=\mu_{\alpha}$ with $\mu_{\alpha} \in N$ for $\alpha=1, \ldots, N$. We assume that

$$
\varphi_{j} \in \mathcal{O}^{q_{\alpha} K_{\alpha}}\left(\hat{S}_{\left(d_{\alpha}+\arg \lambda_{\alpha \beta}+2 n_{\alpha} \pi\right) / q_{\alpha}}\right)
$$


for every $j=0, \ldots, n-1, n_{\alpha}=0, \ldots, \mu_{\alpha}-1, \beta=1, \ldots, l_{\alpha}$ and $\alpha=1, \ldots, N$. Then the normalised formal solution $\hat{u}$ of $(10)$ is $\left(K_{1}, \ldots, K_{N}\right)$-summable in the multidirection $\left(d_{1}, \ldots, d_{N}\right)$.

In general, the sufficient condition for the multisummability of $\hat{u}$ given in Theorem 5 is not necessary, since the multisummability of $\hat{u}$ satisfying (28) does not imply the summability of $\hat{u}_{\alpha \beta \gamma}$ (see Example 2 in the next section). For this reason we define a kind of multisummability for which that implication holds.

Definition 11. Let $\left(d_{1}, \ldots, d_{N}\right)$ be an admissible multidirection with respect to $\left(K_{1}, \ldots, K_{N}\right)$. We say that $\hat{u}$ is $\left(K_{1}, \ldots, K_{N}\right)$-summable in the multidirection $\left(d_{1}, \ldots, d_{N}\right)$ with respect to the decomposition (28) if $\hat{u}_{\alpha \beta \gamma}$ is $K_{\alpha}$-summable in the direction $d_{\alpha}$ (for $\alpha=1, \ldots, N$ ) and is convergent (for $\alpha=N+1, \ldots, \tilde{n}$ ), where $\beta=1, \ldots, l_{\alpha}$ and $\gamma=1, \ldots, n_{\alpha \beta}$.

Now we are ready to prove

Theorem 6. Let $\left(d_{1}, \ldots, d_{N}\right) \in \boldsymbol{R}^{N}$ be an admissible multidirection with respect to $\left(K_{1}, \ldots, K_{N}\right)$ and let $q_{\alpha}=\mu_{\alpha}$ with $\mu_{\alpha} \in N$ for $\alpha=1, \ldots, N$. We assume that $\hat{u}$ is the normalised formal solution of

$$
\left\{\begin{array}{l}
P\left(\partial_{m_{1}, t}, \partial_{m_{2}, z}\right) \hat{u}=0 \\
\partial_{m_{1}, t}^{j} \hat{u}(0, z)=0 \quad(j=0, \ldots, n-2) \\
\partial_{m_{1}, t}^{n-1} \hat{u}(0, z)=\varphi(z) \in \mathcal{O}(D) .
\end{array}\right.
$$

Then $\hat{u}$ is $\left(K_{1}, \ldots, K_{N}\right)$-summable in the multidirection $\left(d_{1}, \ldots, d_{N}\right)$ with respect to the decomposition (28) if and only if

$$
\varphi \in \mathcal{O}^{q_{\alpha} K_{\alpha}}\left(\hat{S}_{\left(d_{\alpha}+\arg \lambda_{\alpha \beta}+2 n_{\alpha} \pi\right) / q_{\alpha}}\right)
$$

for every $n_{\alpha}=0, \ldots, \mu_{\alpha}-1, \beta=1, \ldots, l_{\alpha}$ and $\alpha=1, \ldots, N$.

Proof. The "if part" of the theorem is given immediately by Theorem 4. To prove the "only if" part, we fix $\alpha \in\{1, \ldots, N\}, \beta \in\left\{1, \ldots, l_{\alpha}\right\}$ and we define $\hat{u}_{\alpha \beta}:=\tilde{P}_{\alpha \beta}\left(\partial_{m_{1}, t}, \partial_{m_{2}, z}\right) \hat{u}$, where

$$
\tilde{P}_{\alpha \beta}(\lambda, \zeta):=\tilde{P}(\lambda, \zeta) /\left(\lambda-\lambda_{\alpha \beta}(\zeta)\right)
$$

Since

$$
\hat{\boldsymbol{u}}_{\alpha \beta}=\tilde{\boldsymbol{P}}_{\alpha \beta}\left(\partial_{m_{1}, t}, \partial_{m_{2}, z}\right) \hat{\boldsymbol{u}}=\tilde{\boldsymbol{P}}_{\alpha \beta}\left(\partial_{m_{1}, t}, \partial_{m_{2}, z}\right)\left(\sum_{\gamma=1}^{n_{\alpha \beta}} \hat{\boldsymbol{u}}_{\alpha \beta \gamma}\right)
$$

and $\hat{u}_{\alpha \beta \gamma}$ is $K_{\alpha}$-summable in the direction $d_{\alpha}$ for $\gamma=1, \ldots, n_{\alpha \beta}$, we conclude that $\hat{u}_{\alpha \beta}$ is also $K_{\alpha}$-summable in the direction $d_{\alpha}$. On the other hand, since

$$
\left(\partial_{m_{1}, t}-\lambda_{\alpha \beta}\left(\partial_{m_{2}, z}\right)\right) \tilde{P}_{\alpha \beta}\left(\partial_{m_{1}, t}, \partial_{m_{2}, z}\right)=\tilde{P}\left(\partial_{m_{1}, t}, \partial_{m_{2}, z}\right)
$$


and $\hat{u}_{\alpha \beta}(0, z)=\partial_{m_{1}, t}^{n-1} \hat{u}(0, z)=\varphi(z)$, we see that $\hat{u}_{\alpha \beta}$ satisfies the equation

$$
\left\{\begin{array}{l}
\left(\partial_{m_{1}, t}-\lambda_{\alpha \beta}\left(\partial_{m_{2}, z}\right)\right) \hat{u}_{\alpha \beta}=0 \\
\hat{u}_{\alpha \beta}(0, z)=\varphi(z) \in \mathcal{O}(D) .
\end{array}\right.
$$

Hence by Theorem 4, $\varphi \in \mathcal{O}^{q_{\alpha} K_{\alpha}}\left(\hat{S}_{\left(d_{\alpha}+\arg \lambda_{\alpha \beta}+2 n_{\alpha} \pi\right) / q_{\alpha}}\right)$ for every $n_{\alpha}=0, \ldots, \mu_{\alpha}-1$, which proves the "only if" part of the theorem.

\section{Corrections of the authors previous papers concerning summability of formal solutions of PDEs}

In [15] the author has claimed that the sufficient condition (29) given in Theorem 5 is also necessary for the summability of formal solutions of linear fractional PDEs (see [15, Theorem 4]). This claim is false in a such general statement, as will be seen from the examples below. The correct necessary conditions are given by Theorem 6 .

First, we show that the sufficient condition (29) is not necessary without some additional assumptions on the initial data.

Example 1. Let $\hat{u}$ be a formal solution of

$$
\left\{\begin{array}{l}
\left(\partial_{t}-\partial_{z}^{2}\right)\left(\partial_{t}-\partial_{z}^{3}\right) u=0 \\
u(0, z)=\varphi_{1}(z)=\frac{1}{z-1}+\frac{1}{z-e^{i \pi / 6}}, \\
u_{t}(0, z)=\varphi_{2}(z)=\frac{2}{(z-1)^{3}}-\frac{6}{\left(z-e^{i \pi / 6}\right)^{4}} .
\end{array}\right.
$$

Then $\hat{u}=\hat{u}_{1}+\hat{u}_{2}$, where $\hat{u}_{1}, \hat{u}_{2}$ are the formal solutions of

$$
\begin{array}{ll}
\left(\partial_{t}-\partial_{z}^{2}\right) u_{1}=0 & u_{1}(0, z)=\varphi_{11}(z)=\frac{1}{z-1}, \\
\left(\partial_{t}-\partial_{z}^{3}\right) u_{2}=0 & u_{2}(0, z)=\varphi_{12}(z)=\frac{1}{z-e^{i \pi / 6}},
\end{array}
$$

respectively. Since $\varphi_{11} \in \mathcal{O}^{2}\left(\hat{S}_{\pi / 6} \cup \hat{S}_{\pi / 6+\pi}\right)$ and $\varphi_{12} \in \mathcal{O}^{3 / 2}\left(\hat{S}_{0} \cup \hat{S}_{2 \pi / 3} \cup \hat{S}_{4 \pi / 3}\right)$, by Theorem 4 we conclude that $\hat{u}_{1}$ is 1 -summable in the direction $\pi / 3$ and $\hat{u}_{2}$ is $1 / 2$-summable in the direction 0 . Hence $\hat{u}$ is $(1,1 / 2)$-summable in the multidirection $(\pi / 3,0)$. But $\varphi_{j} \notin \mathcal{O}^{2}\left(\hat{S}_{\pi / 6} \cup \hat{S}_{\pi / 6+\pi}\right)$ and $\varphi_{j} \notin \mathcal{O}^{3 / 2}\left(\hat{S}_{0} \cup \hat{S}_{2 \pi / 3} \cup \hat{S}_{4 \pi / 3}\right)$ for $j=1,2$. It means that the condition (29) is not satisfied. For this reason, in Theorem 6 we additionally assume that the initial data in (10) satisfy condition

$$
\varphi_{j}(z)=0 \quad \text { for } j=0, \ldots, n-2 \quad \text { and } \quad \varphi_{n-1}(z)=\varphi(z) \in \mathcal{O}(D) .
$$


The mistake was made in the proof of [15, Theorem 2 (p. 566)], where the principle of superposition of solutions of linear equations was ill used. This principle may be used only to find the sufficient condition for the analytic continuation (resp. summability) of solutions in terms of the Cauchy data, but can not be used to find the necessary one. For this reason the proof of $[15$, Theorem 2] is correct only under the additional assumption, that the initial data given by $[15$, formula (9)] satisfy condition (30), as in Theorem 6 . Consequently we have to also add the same assumption on the initial data in $[15$, Proposition 9, Theorems 3-4 and Corollary 1] and [16, Theorems 1-2 and Propositions 6, 8-10].

Next, we show that the sufficient condition (29) is not necessary without additional assumptions on the nature of multisummable formal solution.

Example 2. Let $\hat{u}$ be a formal solution of

$$
\left\{\begin{array}{l}
\left(\partial_{t}-\partial_{z}^{2}\right)\left(\partial_{t}-\partial_{z}^{4}\right) u=0 \\
u(0, z)=0 \\
u_{t}(0, z)=\left(\partial_{z}^{4}-\partial_{z}^{2}\right) \varphi(z)
\end{array}\right.
$$

where $\varphi(z):=1 /(1-z)+e^{z^{2}}$. Then $\hat{u}=\hat{u}_{1}+\hat{u}_{2}$, where $\hat{u}_{1}$ and $\hat{u}_{2}$ are the formal solutions of

$$
\left(\partial_{t}-\partial_{z}^{2}\right) u_{1}=0 \quad u_{1}(0, z)=-\varphi(z), \quad\left(\partial_{t}-\partial_{z}^{4}\right) u_{2}=0 \quad u_{2}(0, z)=\varphi(z) .
$$

We will show that $\hat{u}$ is $(1,1 / 3)$-summable in a multidirection $\left(d_{1}, d_{2}\right)$, where $d_{1}, d_{2} \in(0, \pi)$. Since $\varphi \in \mathcal{O}^{2}\left(\hat{S}_{d_{1} / 2} \cup \hat{S}_{d_{1} / 2+\pi}\right)$, by Theorem 4 we conclude that $\hat{u}_{1}$ is 1 -summable in the direction $d_{1}$. Moreover, we have $\hat{u}_{2}=\hat{u}_{21}+\hat{u}_{22}$, where $\hat{u}_{21}$ and $\hat{u}_{22}$ satisfy

$$
\begin{array}{ll}
\left(\partial_{t}-\partial_{z}^{4}\right) u_{21}=0, & u_{21}(0, z)=\varphi_{21}(z)=\frac{1}{1-z} \\
\left(\partial_{t}-\partial_{z}^{4}\right) u_{22}=0, & u_{22}(0, z)=\varphi_{22}(z)=e^{z^{2}} .
\end{array}
$$

Since $\varphi_{21}(z) \in \mathcal{O}^{4 / 3}\left(\hat{S}_{\left(d_{2}+2 n \pi\right) / 4}\right)$ for $n=0,1,2,3$, by Theorem 4 we conclude that $\hat{u}_{21}$ is $1 / 3$-summable in the direction $d_{2}$. Moreover, since $\sum_{n=0}^{\infty} a_{n} z^{n}:=\varphi_{22}(z)=$ $e^{z^{2}} \in \mathcal{O}^{2}(\boldsymbol{C})$ and $\sum_{n=0}^{\infty} a_{n} \Gamma(1+n / 2) z^{n}=\sum_{n=0}^{\infty} z^{2 n}=1 /\left(1-z^{2}\right) \in \mathcal{O}^{4}\left(\hat{\boldsymbol{S}}_{\left(d_{1}+2 n \pi\right) / 4}\right)$ for $n=0,1,2,3$, by [18, Proposition 10] $\hat{u}_{22}$ is 1-summable in the direction $d_{1}$. Hence $\hat{u}$ is $(1,1 / 3)$-summable in the multidirection $\left(d_{1}, d_{2}\right)$, but $\varphi \notin \mathcal{O}^{4 / 3}\left(\hat{S}_{d_{2} / 4} \cup\right.$ $\left.\hat{S}_{\left(d_{2}+2 \pi\right) / 4} \cup \hat{S}_{\left(d_{2}+4 \pi\right) / 4} \cup \hat{S}_{\left(d_{2}+6 \pi\right) / 4}\right)$. It means that the condition (29) is not satisfied. For this reason, in Theorem 6 we additionally assume that $\hat{u}$ is multisummable with respect to the decomposition (28). 
For the same reason, we have to replace in [15, Theorem 4 and Corollary 1] and [17, Corollary 2 and Example] the multisummability of $\hat{u}$ by the multisummability of $\hat{u}$ with respect to the decomposition given by the equation. Moreover, by Example 2, also [17, Remark 6] is false and should be removed.

\section{Inhomogeneous equations}

In the last section we generalise the above results to the inhomogeneous case, where the right-hand side of (10) is replaced by the inhomogeneity $\hat{f}(t, z) \in \boldsymbol{E}[[t]]$. Following [17], without loss of generality we may assume that the Cauchy data $\varphi_{j}$ vanish. So, we consider the following Cauchy problem for general inhomogeneous linear moment partial differential equation with constant coefficients

$$
\left\{\begin{array}{l}
P\left(\partial_{m_{1}, t}, \partial_{m_{2}, z}\right) u=\hat{f} \\
\partial_{m_{1}, t}^{j} u(0, z)=0 \text { for } j=0, \ldots, n-1 .
\end{array}\right.
$$

As in the homogeneous case,

$$
P(\lambda, \zeta)=P_{0}(\zeta) \lambda^{n}-\sum_{j=1}^{n} P_{j}(\zeta) \lambda^{n-j}=P_{0}(\zeta)\left(\lambda^{n}-\sum_{j=1}^{n} \tilde{P}_{j}(\zeta) \lambda^{n-j}\right)
$$

where $P_{0}(\zeta), \ldots, P_{n}(\zeta)$ are polynomials and $\tilde{P}_{j}(\zeta):=P_{j}(\zeta) / P_{0}(\zeta)(j=1, \ldots, n)$ are rational functions.

Using the pseudodifferential operators defined by (9) we have

$$
\begin{aligned}
P\left(\partial_{m_{1}, t}, \partial_{m_{2}, z}\right) & =P_{0}\left(\partial_{m_{2}, z}\right)\left(\partial_{m_{1}, t}^{n}-\sum_{j=1}^{n} \tilde{P}_{j}\left(\partial_{m_{2}, z}\right) \partial_{m_{1}, t}^{n-j}\right) \\
& =: P_{0}\left(\partial_{m_{2}, z}\right) \tilde{P}\left(\partial_{m_{1}, t}, \partial_{m_{2}, z}\right) .
\end{aligned}
$$

Observe that, if $P_{0}\left(\partial_{m_{2}, z}\right) \neq$ const. then the Cauchy problem (31) does not have a unique formal solution. In the homogeneous case this inconvenience was avoiding by the choice of the normalised formal solution. In the inhomogeneous case the formal solution is determined by the formal power series $\hat{g}(t, z) \in \boldsymbol{E}[[t]]$ (see also [8] and [17]), which satisfies the equation

$$
P_{0}\left(\partial_{m_{2}, z}\right) \hat{g}=\hat{f} .
$$

For a given $\hat{g}$ there is exactly one formal solution $\hat{u}$ of the Cauchy problem

$$
\left\{\begin{array}{l}
\tilde{P}\left(\partial_{m_{1}, t}, \partial_{m_{2}, z}\right) \hat{u}=\hat{g} \\
\partial_{m_{1}, t}^{j} \hat{u}(0, z)=0 \text { for } j=0, \ldots, n-1,
\end{array}\right.
$$


which is also a solution of (31) and is called the formal solution of (31) determined by $\hat{g}$.

In the next proposition we find this formal solution $\hat{u}$.

Proposition 6. The formal solution $\hat{u}$ of (31) determined by $\hat{g}$ is given by

$$
\hat{u}(t, z)=\sum_{j=0}^{\infty}\left(\partial_{m_{1}, t}^{-1}\right)^{j+1} q_{j}\left(\partial_{m_{2}, z}\right) \hat{g}(t, z),
$$

where $q_{j}(\zeta)$ is the solution of the difference equation

$$
q_{j}(\zeta)=\sum_{k=1}^{n} \tilde{P}_{k}(\zeta) q_{j-k}(\zeta) \quad \text { for } j \geq n
$$

with the initial conditions $q_{0}(\zeta)=\cdots=q_{n-2}(\zeta)=0$ and $q_{n-1}(\zeta)=1$.

Proof. Since $q_{j}(\zeta)$ is a rational function, the pseudodifferential operator $q_{j}\left(\partial_{m_{2}, z}\right)$ is well-defined.

To finish the proof, it is sufficient to show that the formal series given by (33) is a solution of (32). To this end observe that $\partial_{m_{1}, t}^{j} \hat{u}(0, z)=0$ for $j=$ $0, \ldots, n-1$ and

$$
\begin{aligned}
\tilde{\boldsymbol{P}}\left(\partial_{m_{1}, t}, \partial_{m_{2}, z}\right) \hat{\boldsymbol{u}}(t, z) & \tilde{\boldsymbol{P}}\left(\partial_{m_{1}, t}, \partial_{m_{2}, z}\right)\left(\sum_{j=n-1}^{\infty}\left(\partial_{m_{1}, t}^{-1}\right)^{j+1} q_{j}\left(\partial_{m_{2}, z}\right) \hat{\boldsymbol{g}}(t, z)\right) \\
= & \sum_{j=n-1}^{\infty}\left(\partial_{m_{1}, t}^{-1}\right)^{j-n+1} q_{j}\left(\partial_{m_{2}, z}\right) \hat{\boldsymbol{g}}(t, z) \\
& -\sum_{k=1}^{n} \sum_{j=n-1}^{\infty}\left(\partial_{m_{1}, t}^{-1}\right)^{j-n+1+k} \tilde{P}_{k}\left(\partial_{m_{2}, z}\right) q_{j}\left(\partial_{m_{2}, z}\right) \hat{g}(t, z) \\
= & \sum_{j=n-1}^{\infty}\left(\partial_{m_{1}, t}^{-1}\right)^{j-n+1} q_{j}\left(\partial_{m_{2}, z}\right) \hat{\boldsymbol{g}}(t, z) \\
& -\sum_{j=n}^{\infty} \sum_{k=1}^{n}\left(\partial_{m_{1}, t}^{-1}\right)^{j-n+1} \tilde{\boldsymbol{P}}_{k}\left(\partial_{m_{2}, z}\right) q_{j-k}\left(\partial_{m_{2}, z}\right) \hat{\boldsymbol{g}}(t, z) \\
= & \sum_{j=n-1}^{\infty}\left(\partial_{m_{1}, t}^{-1}\right)^{j-n+1} q_{j}\left(\partial_{m_{2}, z}\right) \hat{g}(t, z)-\sum_{j=n}^{\infty}\left(\partial_{m_{1}, t}^{-1}\right)^{j-n+1} q_{j}\left(\partial_{m_{2}, z}\right) \hat{g}(t, z) \\
= & q_{n-1}\left(\partial_{m_{2}, z}\right) \hat{g}(t, z)=\hat{g}(t, z) .
\end{aligned}
$$


Now we consider the simple inhomogeneous moment pseudodifferential equation

$$
\left\{\begin{array}{l}
\left(\partial_{m_{1}, t}-\lambda\left(\partial_{m_{2}, z}\right)\right)^{\beta} u(t, z)=\hat{g}(t, z) \\
\partial_{m_{1}, t}^{j} u(0, z)=0, \quad j=0, \ldots, \beta-1
\end{array}\right.
$$

We show that

Lemma 8. The formal solution $\hat{u}$ of (34) is given by

$$
\hat{u}(t, z)=\sum_{j=\beta-1}^{\infty}\left(\begin{array}{c}
j \\
\beta-1
\end{array}\right)\left(\partial_{m_{1}, t}^{-1}\right)^{j+1} \lambda^{j-\beta+1}\left(\partial_{m_{2}, z}\right) \hat{g}(t, z) .
$$

Proof. Let $\hat{u}$ be given by (35). Then

$$
\partial_{m_{1}, t}^{n} \hat{u}(t, z)=\sum_{j=\beta-1}^{\infty}\left(\begin{array}{c}
j \\
\beta-1
\end{array}\right)\left(\partial_{m_{1}, t}^{-1}\right)^{j+1-n} \lambda^{j-\beta+1}\left(\partial_{m_{2}, z}\right) \hat{g}(t, z)
$$

It means that $\partial_{m_{1}, t}^{n} \hat{u}(0, z)=0$ for $n=0, \ldots, \beta-1$.

Observe that

$$
\begin{aligned}
\left(\partial_{m_{1}, t}\right. & \left.-\lambda\left(\partial_{m_{2}, z}\right)\right) \sum_{j=0}^{\infty}\left(\partial_{m_{1}, t}^{-1}\right)^{j+1} \lambda^{j}\left(\partial_{m_{2}, z}\right) \hat{g}(t, z) \\
& =\sum_{j=0}^{\infty}\left(\partial_{m_{1}, t}^{-1}\right)^{j} \lambda^{j}\left(\partial_{m_{2}, z}\right) \hat{g}(t, z)-\sum_{j=0}^{\infty}\left(\partial_{m_{1}, t}^{-1}\right)^{j+1} \lambda^{j+1}\left(\partial_{m_{2}, z}\right) \hat{g}(t, z)=\hat{g}(t, z)
\end{aligned}
$$

Moreover, if $n \geq 1$ then by (13) we have

$$
\begin{aligned}
\left(\partial_{m_{1}, t}\right. & \left.-\lambda\left(\partial_{m_{2}, z}\right)\right) \sum_{j=n}^{\infty}\left(\begin{array}{c}
j \\
n
\end{array}\right)\left(\partial_{m_{1}, t}^{-1}\right)^{j+1} \lambda^{j-n}\left(\partial_{m_{2}, z}\right) \\
& =\sum_{j=n-1}^{\infty}\left(\begin{array}{c}
j+1 \\
n
\end{array}\right)\left(\partial_{m_{1}, t}^{-1}\right)^{j+1} \lambda^{j+1-n}\left(\partial_{m_{2}, z}\right)-\sum_{j=n}^{\infty}\left(\begin{array}{l}
j \\
n
\end{array}\right)\left(\partial_{m_{1}, t}^{-1}\right)^{j+1} \lambda^{j+1-n}\left(\partial_{m_{2}, z}\right) \\
& =\sum_{j=n-1}^{\infty}\left(\begin{array}{c}
j \\
n-1
\end{array}\right)\left(\partial_{m_{1}, t}^{-1}\right)^{j+1} \lambda^{j+1-n}\left(\partial_{m_{2}, z}\right) .
\end{aligned}
$$


Consequently

$$
\begin{aligned}
\left(\partial_{m_{1}, t}-\lambda\left(\partial_{m_{2}, z}\right)\right)^{\beta} \sum_{j=\beta-1}^{\infty}\left(\begin{array}{c}
j \\
\beta-1
\end{array}\right)\left(\partial_{m_{1}, t}^{-1}\right)^{j+1} \lambda^{j-\beta+1}\left(\partial_{m_{2}, z}\right) \hat{g}(t, z) \\
=\left(\partial_{m_{1}, t}-\lambda\left(\partial_{m_{2}, z}\right)\right)^{\beta-1} \sum_{j=\beta-2}^{\infty}\left(\begin{array}{c}
j \\
\beta-2
\end{array}\right)\left(\partial_{m_{1}, t}^{-1}\right)^{j+1} \lambda^{j-\beta+2}\left(\partial_{m_{2}, z}\right) \hat{g}(t, z) \\
=\cdots=\left(\partial_{m_{1}, t}-\lambda\left(\partial_{m_{2}, z}\right)\right) \sum_{j=0}^{\infty}\left(\partial_{m_{1}, t}^{-1}\right)^{j+1} \lambda^{j}\left(\partial_{m_{2}, z}\right) \hat{g}(t, z)=\hat{g}(t, z)
\end{aligned}
$$

and the proof is complete.

Next, using Proposition 6 and the factorisation of operator $\tilde{P}\left(\partial_{m_{1}, t}, \partial_{m_{2}, z}\right)$, we obtain the following decomposition of solution of (31) determined by $\hat{g}$.

Theorem 7. Let $\hat{u}$ be the formal solution of (31) determined by $\hat{g}$ and

$$
\tilde{P}\left(\partial_{m_{1}, t}, \partial_{m_{2}, z}\right)=\left(\partial_{m_{1}, t}-\lambda_{1}\left(\partial_{m_{2}, z}\right)\right)^{n_{1}} \ldots\left(\partial_{m_{1}, t}-\lambda_{l}\left(\partial_{m_{2}, z}\right)\right)^{n_{l}} .
$$

Then $\hat{u}=\sum_{\alpha=1}^{l} \sum_{\beta=1}^{n_{\alpha}} \hat{u}_{\alpha \beta}$, where $\hat{u}_{\alpha \beta}$ is a formal solution of

$$
\left\{\begin{array}{l}
\left(\partial_{m_{1}, t}-\lambda_{\alpha}\left(\partial_{m_{2}, z}\right)\right)^{\beta} \hat{u}_{\alpha \beta}(t, z)=\hat{g}_{\alpha \beta}(t, z) \\
\partial_{m_{1}, t}^{j} \hat{u}_{\alpha \beta}(0, z)=0 \text { for } j=0, \ldots, \beta-1
\end{array}\right.
$$

and $\hat{g}_{\alpha \beta}(t, z)=d_{\alpha \beta}\left(\partial_{m_{2}, z}\right) \hat{g}(t, z)$ for some holomorphic function $d_{\alpha \beta}(\zeta)$ of polynomial growth.

Proof. By Proposition 6 the formal solution of (31) determined by $\hat{g}$ is given by

$$
\hat{u}(t, z)=\sum_{j=0}^{\infty}\left(\partial_{m_{1}, t}^{-1}\right)^{j+1} q_{j}\left(\partial_{m_{2}, z}\right) \hat{g}(t, z),
$$

where

$$
q_{j}(\zeta)=\sum_{\alpha=1}^{l} \sum_{\beta=1}^{\min \left\{j+1, n_{\alpha}\right\}} c_{\alpha \beta}(\zeta) \frac{j !}{(j+1-\beta) !} \lambda_{\alpha}^{j}(\zeta),
$$

$c_{\alpha \beta}(\zeta)$ is a holomorphic function of polynomial growth for sufficiently large $|\zeta|$ (see Section 5 in [15] for more details) and $\lambda_{\alpha}(\zeta)$ is the root of characteristic equation with multiplicity $n_{\alpha}$.

It means that $\hat{u}=\sum_{\alpha=1}^{l} \sum_{\beta=1}^{n_{\alpha}} \hat{u}_{\alpha \beta}$, where

$$
\hat{u}_{\alpha \beta}=\sum_{j=\beta-1}^{\infty} \frac{j !}{(j+1-\beta) !}\left(\partial_{m_{1}, t}^{-1}\right)^{j+1} \lambda_{\alpha}^{j}\left(\partial_{m_{2}, z}\right) c_{\alpha \beta}\left(\partial_{m_{2}, z}\right) \hat{g}(t, z) .
$$


By Lemma $8, \hat{u}_{\alpha \beta}$ is a formal solution of (36) with

$$
\hat{g}_{\alpha \beta}(t, z)=d_{\alpha \beta}\left(\partial_{m_{2}, z}\right) \hat{g}(t, z) \quad \text { and } \quad d_{\alpha \beta}(\zeta)=(\beta-1) ! c_{\alpha \beta}(\zeta) \lambda_{\alpha}^{\beta-1}(\zeta) .
$$

Now we are ready to study the Gevrey order of formal solution to inhomogeneous equation. First we consider the simple equation (34). We have

Proposition 7. Let $\hat{u}$ be the formal solution of (34) determined by a Gevrey series $\hat{g} \in \boldsymbol{E}[[t]]_{s}$ of order $s \geq 0$ and let $q>0$ be a pole order of $\lambda(\zeta)$. Then $\hat{u}$ is a Gevrey series of order $\max \left\{\left(q k_{1}-k_{2}\right) /\left(k_{1} k_{2}\right), s\right\}$ with respect to $t$.

Proof. Since

$$
\hat{g}(t, z)=\sum_{n=0}^{\infty} \frac{g_{n}(z)}{m_{1}(n)} t^{n} \quad \text { with } g_{n}(z) \in \boldsymbol{E}(r)
$$

is a Gevrey series of order $s \geq 0$, there exist $A, B>0$ such that

$$
\max _{|z| \leq r}\left|g_{n}(z)\right| \leq A B^{n} \Gamma\left(1+\left(s+1 / k_{1}\right) n\right) \quad \text { for } n=0,1, \ldots
$$

By Lemma 8 we have

$$
\begin{aligned}
\hat{u}(t, z) & =\sum_{j=\beta-1}^{\infty}\left(\begin{array}{c}
j \\
\beta-1
\end{array}\right)\left(\partial_{m_{1}, t}^{-1}\right)^{j+1} \lambda^{j-\beta+1}\left(\partial_{m_{2}, z}\right) \hat{g}(t, z) \\
& =\sum_{j=\beta-1}^{\infty}\left(\begin{array}{c}
j \\
\beta-1
\end{array}\right) \lambda^{j-\beta+1}\left(\partial_{m_{2}, z}\right) \sum_{n=0}^{\infty} \frac{g_{n}(z)}{m_{1}(n+j+1)} t^{n+j+1} \\
& \stackrel{k=n+j+1}{=} \sum_{k=\beta}^{\infty} \frac{t^{k}}{m_{1}(k)} \sum_{j=\beta-1}^{k-1}\left(\begin{array}{c}
j \\
\beta-1
\end{array}\right) \lambda^{j-\beta+1}\left(\partial_{m_{2}, z}\right) g_{k-j-1}(z) \\
& =: \sum_{k=\beta}^{\infty} \frac{u_{k}(z) t^{k}}{m_{1}(k)} .
\end{aligned}
$$

Now, by Lemma 1 and by (37), we obtain the estimate

$$
\begin{aligned}
\left|u_{k}(z)\right| & \leq \sum_{j=\beta-1}^{k-1}\left(\begin{array}{c}
j \\
\beta-1
\end{array}\right)\left|\lambda^{j-\beta+1}\left(\partial_{m_{2}, z}\right) g_{k-j-1}(z)\right| \\
& \leq \tilde{A} \tilde{B}^{k} \sum_{j=\beta-1}^{k-1} \Gamma\left(1+j q / k_{2}\right) \Gamma\left(1+(k-j-1)\left(s+1 / k_{1}\right)\right)
\end{aligned}
$$


for $z \in D_{r}$. Hence there exist $C, D<\infty$ such that

$$
\sup _{|z|<r}\left|u_{k}(z)\right| \leq C D^{K} \Gamma\left(1+k\left(\tilde{s}+1 / k_{1}\right)\right) \quad \text { for } k=0,1, \ldots,
$$

where $\tilde{s}=\max \left\{\left(q k_{1}-k_{2}\right) /\left(k_{1} k_{2}\right), s\right\}$.

By Theorem 7 and Proposition 7, we obtain the Gevrey estimates for solutions of (31), which improve the result of Balser and Yoshino [8]. Namely we have

Theorem 8. Let $\hat{u}$ be a formal solution of (31) determined by a Gevrey series $\hat{g} \in \boldsymbol{E}[[t]]_{s}$ of order $s \geq 0, \hat{u}=\sum_{\alpha=1}^{l} \sum_{\beta=1}^{n_{\alpha}} \hat{u}_{\alpha \beta}$ be the decomposition of solution constructed in Theorem 7 and let $q_{\alpha}>0$ be a pole order of $\lambda_{\alpha}(\zeta)$. Then $\hat{u}_{\alpha \beta}$ is a Gevrey series of order $\max \left\{\left(q_{\alpha} k_{1}-k_{2}\right) /\left(k_{1} k_{2}\right), s\right\}$ with respect to $t$.

One can also generalise Theorem 3 and Theorem 4 to the inhomogeneous equation (34). Unfortunately, in the inhomogeneous case we have to replace the integral representation (20) of solution by the power series representation given by (35). Using this last representation we immediately obtain the characterisation of the analytic continuation properties (in case $1 / k_{1}=q / k_{2}$ ) and summability (in case $1 / k_{1}<q / k_{2}$ ) of solution of (34) in terms of inhomogeneity $\hat{g}$.

\section{Acknowledgement}

The author is grateful to the anonymous referee for the careful reading and the valuable comments and suggestions to improve this paper.

\section{References}

[1] Balser, W., Divergent solutions of the heat equation: on an article of Lutz, Miyake and Schäfke, Pacific J. Math., 188 (1999), 53-63.

[2] Balser, W., Formal power series and linear systems of meromorphic ordinary differential equations, Springer-Verlag, New York, 2000.

[3] Balser, W., Multisummability of formal power series solutions of partial differential equations with constant coefficients, J. Differential Equations, 201 (2004), 63-74.

[4] Balser, W., Power series solutions of the inhomogeneous heat equation, Recent trends in Microlocal Analysis, Sūrikaisekikenkyūsho Kōkyūroku, No. 1412, 2005, pp. 151-159.

[ 5 ] Balser, W., Duval, A. and Malek, S., Summability of formal solutions for abstract Cauchy problems and related convolution equations, Ulmer Seminare, 11 (2006), 29-44.

[6] Balser, W. and Loday-Richaud, M., Summability of solutions of the heat equation with inhomogeneous thermal conductivity in two variables, Adv. Dyn. Syst. Appl., 4 (2009), 159177.

[7] Balser, W. and Miyake, M., Summability of formal solutions of certain partial differential equations, Acta Sci. Math. (Szeged), 65 (1999), 543-551. 
[ 8 ] Balser, W. and Yoshino, M., Gevrey order of formal power series solutions of inhomogeneous partial differential equations with constant coefficients, Funkcial. Ekvac., 53 (2010), 411-434.

[9] Ichinobe, K., Integral representation for Borel sum of divergent solution to a certain nonKovalevski type equation, Publ. Res. Inst. Math. Sci., 39 (2003), 657-693.

[10] Lutz, D. A., Miyake, M. and Schäfke, R., On the Borel summability of divergent solutions of the heat equation, Nagoya Math. J., 154 (1999), 1-29.

[11] Łysik, G., Borel summable solutions of the Burgers equation, Ann. Pol. Math., 95 (2009), 187-197.

[12] Malek, S., On the summability of formal solutions of linear partial differential equations, J. Dyn. Control Syst., 11 (2005), 389-403.

[13] Michalik, S., Summability of divergent solutions of the $n$-dimensional heat equation, J. Differential Equations, 229 (2006), 353-366.

[14] Michalik, S., Summability of formal solutions to the $n$-dimensional inhomogeneous heat equation, J. Math. Anal. Appl., 347 (2008), 323-332.

[15] Michalik, S., On the multisummability of divergent solutions of linear partial differential equations with constant coefficients, J. Differential Equations, 249 (2010), 551-570.

[16] Michalik, S., Summability and fractional linear partial differential equations, J. Dyn. Control Syst., 16 (2010), 557-584.

[17] Michalik, S., Multisummability of formal solutions of inhomogeneous linear partial differential equations with constant coefficients, J. Dyn. Control Syst., 18 (2012), 103-133.

[18] Michalik, S., Summability of formal solutions of linear partial differential equations with divergent initial data, Manuscript (available on the web page http://www.impan.pl/ slawek/ divergent.pdf), 2012.

[19] Miyake, M., Borel summability of divergent solutions of the Cauchy problem to nonKovaleskian equations, Partial Differential Equations and Their Applications (Wuhan, 1999), World Sci. Publ., River Edge, NJ, 1999, pp. 225-239.

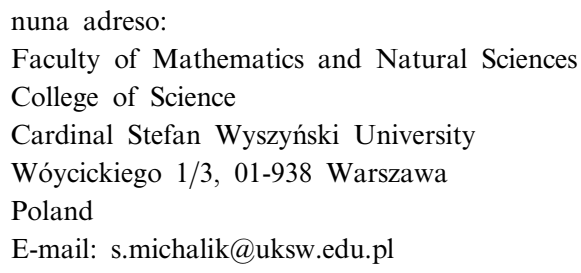

(Ricevita la 13-an de decembro, 2011)

(Reviziita la 28-an de julio, 2012) 